\title{
The Great Slump: Mrk 926 reveals discrete and varying Balmer line satellite components during a drastic phase of decline $\star \star \star$
}

\author{
Wolfram Kollatschny ${ }^{1}$, Martin W. Ochmann ${ }^{1,2}$, Shai Kaspi ${ }^{3}$, Claas Schumacher ${ }^{1}$, Ehud Behar ${ }^{4}$, Doron Chelouche ${ }^{5}$ \\ Keith Horne ${ }^{6}$, Björn Müller ${ }^{1,7}$, Stephen E. Rafter ${ }^{4}$, Rolf Chini ${ }^{2,8}$, Martin Haas ${ }^{2}$ and Malte A. Probst ${ }^{1}$ \\ ${ }^{1}$ Institut für Astrophysik, Universität Göttingen, Friedrich-Hund Platz 1, D-37077 Göttingen, Germany \\ e-mail: wkollat@astro.physik.uni-goettingen.de \\ 2 Astronomisches Institut, Ruhr-Universität Bochum, Universitätsstrasse 150, 44801 Bochum, Germany \\ 3 School of Physics \& Astronomy and the Wise Observatory, The Raymond and Beverly Sackler Faculty of Exact Sciences Tel-Aviv \\ University, Tel-Aviv 69978, Israel \\ 4 Technion, Institute of Technology, Haifa 3200003, Israel \\ 5 Physics Department and the Haifa Research Center for Theoretical Physics and Astrophysics, University of Haifa, Haifa 3498838, \\ Israel \\ ${ }^{6}$ SUPA School of Physics and Astronomy, University of St. Andrews, St. Andrews, Fife, KY16 9SS, UK \\ 7 Max-Planck-Institut für Sonnensystemforschung, Justus-von-Liebig-Weg 3, D-37077 Göttingen, Germany \\ 8 Instituto de Astronomía, Universidad Católica del Norte, Avenida Angamos 0610, Antofagasta, Chile
}

Received August 12, 2021 ; Accepted October 11, 2021

\begin{abstract}
Aims. Mrk 926 is known to be a highly variable active galactic nucleus. Furthermore, it is known to show very broad-line profiles We intended to study the continuum and line profile variations of this object with high temporal resolution in order to determine its broad-line region (BLR) structure and to derive its black hole mass.

Methods. We carried out a high-cadence spectroscopic variability campaign of Mrk 926 with the 10m HET telescope, aided by photometric V-band data taken with the C18 telescope at the Wise Observatory, over a period of about five months. We extracted spectroscopic continuum and line light curves, and computed cross-correlation functions (CCFs) as well as velocity-resolved CCFs with respect to the combined spectroscopic and photometric V-band light curve.

Results. The continuum luminosity of Mrk 926 showed a drastic decrease during our campaign. The luminosity dropped to less than $50 \%$ of its original luminosity within only 2.5 months. Furthermore, the spectra of Mrk 926 show complex and very broad Balmer line profiles, including outer Balmer satellites ranging from \pm 5000 to $\pm 13000 \mathrm{~km} \mathrm{~s}^{-1}$. The integrated $\mathrm{H} \alpha, \mathrm{H} \beta$, and $\mathrm{He}$ I $\lambda 5876$ line light curves are delayed relative to the continuum light curve. The $\mathrm{H} \alpha$ and $\mathrm{H} \beta$ lines show two velocity-delay structures in the central part of their line profile (within $\pm 5000 \mathrm{~km} \mathrm{~s}^{-1}$ ), at $\sim 10$ and $\sim 57$ light-days and at $\sim 5$ and $\sim 48$ light-days, respectively. These structures might be interpreted as the upper and lower halves of an ellipse in the velocity-delay plane, which might be the signature of a lineemitting ring, inclined by $\sim 50^{\circ}$ to the line of sight and orbiting the black hole at radii, $R$, of 33.5 and 26.5 light-days. We determined continuum luminosities, $\log \left(\lambda L_{\lambda} / \mathrm{erg} \mathrm{s}^{-1}\right)$, of 43.68 to 44.13 , which are in good agreement with the established $R_{\mathrm{BLR}}-L_{\mathrm{AGN}}$ relation. Adopting delays of 33.5 and 26.5 days for $\mathrm{H} \alpha$ and $\mathrm{H} \beta$, respectively, we derive a black hole mass of $(1.1 \pm 0.2) \times 10^{8} M_{\odot}$; this indicates a low Eddington ratio, which decreased from 8 to 3 percent during our campaign. The Balmer satellite components show a higher correlation coefficient with respect to the continuum than the central line profile, and their response to the continuum variations is on the order of only $3-5$ days. We attribute this to the central line segment and the Balmer satellites having different, spatially distinct regions of origin.
\end{abstract}

Key words. Galaxies: active - Galaxies: Seyfert - Galaxies: nuclei - Galaxies: individual: Mrk926 - (Galaxies:) quasars: emission lines

\section{Introduction}

Active galactic nuclei (AGN) are variable in all frequency bands on timescales of days to years. It is generally accepted that the variability of the central ionizing continuum source causes line intensity variations in the broad emission lines. Furthermore, changes in the kinematics and structure in the broadline region (BLR) as well as obscuration can induce variations in the line profiles. However, many details of the innermost

\footnotetext{
* Based on observations obtained with the Hobby-Eberly Telescope. $\star \star$ The reduced spectra and the photometric light curve are only available in electronic form at the CDS via anonymous ftp to cdsarc. u-strasbg.fr (130.79.128.5) or via http://cdsweb.u-strasbg. $\mathrm{fr} / \mathrm{cgi}-\mathrm{bin} / \mathrm{qcat}$ ? J/A+A/.
}

BLR, such as size, structure, and kinematics, are poorly understood. In addition, most of the variability campaigns carried out in the past were devoted to Seyfert galaxies that show broad emission lines with full widths at half maximum (FWHMs) of 1000 to $4000 \mathrm{~km} \mathrm{~s}^{-1}$, and only very few reverberation campaigns have been carried out for AGN with very broad lines (FWHM $\gg 4000 \mathrm{~km} \mathrm{~s}^{-1}$; e.g., 3C390.3: Sergeev et al. 2002; Gezari et al. 2007, and other AGN therein). Broad-line reverberation studies exist for more than 50 Seyfert galaxies (e.g., Peterson et al. 2002; Kaspi et al. 2005; Denney et al. 2009; Barth et al. 2011; Bentz et al. 2013; Shapovalova et al. 2013; Shen et al. 2016; Du et al. 2018, and references therein). However, detailed velocity-resolved reverberation mapping studies have been car- 
ried out for only about a dozen Seyfert galaxies (e.g., Mrk 110: Kollatschny (2003); Arp 151: Bentz et al. (2008); Mrk 335, Mrk 1501, 3C 120, PG 2130: Grier et al. (2013b); 3C120: Kollatschny et al. (2014); Mrk 335, Mrk 1044, Mrk 142: Xiao et al. (2018); HE1136-2304: Kollatschny et al. (2018); NGC 5548: Horne et al. (2021) and references therein).

Mrk 926 can be classified as either a strong Seyfert 1 galaxy or a quasar based on its luminosity during the campaign presented here (see Sect. 4.4). Its Balmer and He I lines have widths (FWHM) of more than $5000 \mathrm{~km} \mathrm{~s}^{-1}$. In a study on the long-term variability of very broad-line AGN (Kollatschny et al. 2006), Mrk 926 additionally exhibited strong $\mathrm{H} \beta$ line variability amplitudes. The nuclear activity in Mrk 926 was detected by Ward et al. (1978) when they took a spectrum of the optical counterpart of a strong X-ray source. Mrk 926 is a compact source on direct images in the optical (Garnier et al. 1996). We show an SDSS (DR16: Ahumada et al. 2020) image of Mrk 926 in Fig. 1. Based on SDSS r-band images, it has an isophotal diameter (major axis) of 57.12 arcsec. This corresponds to $53 \mathrm{kpc}$. The deVaucouleurs diameter amounts to 6.00 arcsec, corresponding to 5.56 $\mathrm{kpc}^{1}$. Mrk 926 is the brighter galaxy member (R.A. = 23:04:43.5, Dec. $=-08: 41: 09(2000), z=0.04701)$ in a double system with PGC 1000273. This close starburst companion is located one arcmin to the south.

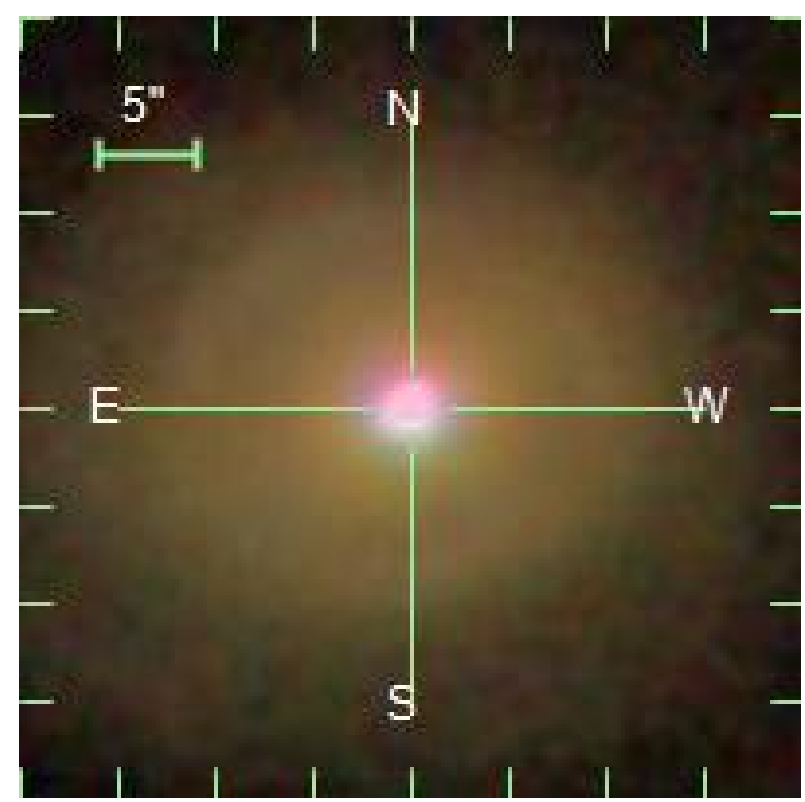

Fig. 1: Composite SDSS image of Mrk 926 taken from the SDSS SkyServer ${ }^{2}$. The crosshairs are centered on the nucleus.

Osterbrock \& Shuder (1982) published the first emissionline profiles of Mrk 926. They reported that the lines did not vary between July 1978 and December 1979 . We were able to show that Mrk 926 varied by a factor of 2 in the continuum as well as by a factor of 2.5 in the Balmer lines over a period of eight years between 1990 and 1997 (Kollatschny et al. 2006). In a follow-up paper (Kollatschny \& Zetzl 2010), we focused on line profile variations in Mrk 926 as the line profiles of AGN and their variations can give us information about the structure of the line-emitting region. We intensively studied the variations in individual line segments of the Balmer lines and the changes

\footnotetext{
1 https://ned.ipac.caltech.edu/

2 https://skyserver.sdss.org
}

in the profiles during a variability campaign in 2005 and another shorter campaign conducted in 2004.

Here, we selected Mrk 926 (MCG-2-58-22) as a target for an even more detailed, combined spectroscopic and photometric variability study of an AGN that shows very broad emission lines. The paper is structured as follows: In Sect. 2 we describe the observations and data reduction. The results of the variability and reverberation mapping study are presented in Sect. 3. We discuss the results in Sect. 4 and give a summary in Sect. 5. Throughout this paper, we assume a $\Lambda$ cold dark matter cosmology with a Hubble constant of $H_{0}=73 \mathrm{~km} \mathrm{~s}^{-1} \mathrm{Mpc}^{-1}$, $\Omega_{\Lambda}=0.73$, and $\Omega_{\mathrm{M}}=0.27$. With a redshift of $z=0.04701$, this results in a luminosity distance of $D_{\mathrm{L}}=200.2 \mathrm{Mpc}=6.18$ $\times 10^{26} \mathrm{~cm}$ using the cosmology calculator developed by Wright (2006).

\section{Observations and data reduction}

\subsection{Spectroscopic observations with HET}

We took optical spectra of Mrk 926 with the 9.2 m Hobby-Eberly Telescope (HET) located at the McDonald Observatory at 31 epochs between 2010 July 19 and November 19. Thus, we obtained 31 spectra over a period of 122.7 days. The date of the campaign was fixed by the allocated observing time slots. The average interval between the observations was 4.1 days. Excluding the first two observations, the average time interval between observations reduces to 2.7 days with a majority of consecutive observations only 1-2 days apart. The log of our spectroscopic observations is given in Table 1. All spectroscopic observations were taken with identical instrumental setups. We used the Marcario Low Resolution Spectrograph (LRS) attached at the prime focus of HET. The detector was a 3072x1024 $15 \mu \mathrm{m}$ pixel Ford Aerospace charge-coupled device (CCD) with $2 \times 2$ binning. The spectra cover the wavelength range from $4160 \AA$ to $6930 \AA$ (LRS grism 2 configuration) in the rest frame of the galaxy (and $4350 \AA$ to $7250 \AA$ observed frame) with a resolving power of 650 at $5000 \AA$ ( $7.7 \AA$ FWHM). Nearly all observations were taken with an exposure time of 20 minutes, which in most cases yielded a $\mathrm{S} / \mathrm{N}$ of $>100$ per resolution element in the continuum. The slit width was fixed to 2 '.0 projected on the sky at an optimized position angle to minimize differential refraction. Furthermore, all observations were taken at the same airmass owing to the particular design of the HET. Typical seeing values ranged from 1 ".4 to 1 '.9.

In addition to the spectra of Mrk 926, necessary calibration images including bias, flat, and arc frames ( $\mathrm{CdHgAr}$ and $\mathrm{Xe}$ ), as well as spectrophotometric standard stars (Hiltner 600) for flux calibration were taken. We reduced the spectra (bias subtraction, cosmic ray correction, flat-field correction, 2D-wavelength calibration, night sky subtraction, flux calibration, etc.) in a homogeneous way with standard IRAF ${ }^{3}$ reduction packages (Tody 1986, 1993) like we did in previous publications (e.g., Kollatschny et al. 2001). In addition, we corrected for atmospheric absorption in the oxygen B band by fitting the continuum of the standard star Hiltner 600 and using this fit to normalize its spectrum. In this way, we created a template of the oxygen absorption that we scaled to each object spectrum individually. The spatial resolution per binned pixel was 0.472 . We extracted four columns in our object spectra. This conforms to the slit width. Great care was taken to ensure high-quality intensity and wavelength calibrations to keep the intrinsic measurement errors very

${ }^{3}$ https://iraf.net/ 
low (e.g., Kollatschny et al. 2001; Kollatschny \& Zetzl 2010). The spectra were not always taken under photometric conditions. Therefore, we calibrated all spectra to the same absolute forbidden narrow-line fluxes under the assumption of a non-varying narrow-line flux on timescales of years. We chose [O III] $\lambda 4959$ and [O III] $\lambda 5007$, as well as [O I] $\lambda 6300 / 63$, [N $\mathrm{NI}_{\mathrm{II}} \lambda \lambda 6548 / 84$, and [S II] $\lambda 6717 / 31$ as calibration lines for the blue and red section of the spectrum, respectively. The blue part of the spectrum was scaled to an [O III] $\lambda 5007$ flux of $3.14 \times 10^{-13} \mathrm{erg} \mathrm{s}^{-1} \mathrm{~cm}^{-2}$. The same flux value has been used before for Mrk 926 in Kollatschny et al. (2006) and Kollatschny \& Zetzl (2010). Durret \& Bergeron (1988) and Morris \& Ward (1988) derived similar [O III] 25007 fluxes from their Mrk926 spectra: $2.05 \times 10^{-13} \mathrm{erg} \mathrm{s}^{-1} \mathrm{~cm}^{-2}$ and $3.31 \times 10^{-13} \mathrm{erg} \mathrm{s}^{-1} \mathrm{~cm}^{-2}$, respectively. We note that hereafter all continuum flux densities and line fluxes at wavelengths $>6500 \AA$ (observed frame) refer to the intercalibration with respect to [O I ] $\lambda 6300 / 63,\left[\mathrm{~N}_{\text {II }}\right] \lambda 6548 / 84$, and [S II] $\lambda 6717 / 31$. The accuracy of the flux calibration was tested for all forbidden emission lines in the spectra. We calculated difference spectra for all epochs with respect to the mean spectrum of our variability campaign. Corrections for small spectral shifts $(<0.5 \AA)$ and small scaling factors were implemented by minimizing the residuals of the narrow emission lines in the difference spectra. A relative flux accuracy of $1-2 \%$ was obtained for most of our spectra.

Table 1: Log of spectroscopic HET observations of Mrk 926.

\begin{tabular}{|c|c|c|}
\hline Mod. Julian Date & UT Date & $\begin{array}{l}\text { Exp. time } \\
\text { [sec.] }\end{array}$ \\
\hline 55396.41 & 2010-07-19 & 1200 \\
\hline 55397.42 & $2010-07-20$ & 1200 \\
\hline 55441.31 & 2010-09-02 & 1200 \\
\hline 55443.30 & 2010-09-04 & 1200 \\
\hline 55444.30 & $2010-09-05$ & 1200 \\
\hline 55445.28 & 2010-09-06 & 1200 \\
\hline 55446.29 & 2010-09-07 & 1200 \\
\hline 55451.27 & 2010-09-12 & 1200 \\
\hline 55452.26 & $2010-09-13$ & 1200 \\
\hline 55453.26 & 2010-09-14 & 1200 \\
\hline 55467.23 & $2010-09-28$ & 1800 \\
\hline 55470.20 & 2010-10-01 & 1200 \\
\hline 55471.21 & $2010-10-02$ & 1200 \\
\hline 55472.22 & $2010-10-03$ & 1200 \\
\hline 55477.20 & $2010-10-08$ & 1200 \\
\hline 55480.20 & 2010-10-11 & 1800 \\
\hline 55481.20 & 2010-10-12 & 951 \\
\hline 55483.20 & $2010-10-14$ & 1300 \\
\hline 55494.16 & $2010-10-25$ & 1200 \\
\hline 55496.15 & $2010-10-27$ & 1200 \\
\hline 55497.16 & $2010-10-28$ & 1200 \\
\hline 55498.17 & $2010-10-29$ & 1200 \\
\hline 55499.16 & $2010-10-30$ & 1200 \\
\hline 55501.14 & 2010-11-01 & 1200 \\
\hline 55505.12 & 2010-11-05 & 1200 \\
\hline 55506.12 & 2010-11-06 & 1200 \\
\hline 55507.11 & 2010-11-07 & 1200 \\
\hline 55508.11 & 2010-11-08 & 1200 \\
\hline 55514.12 & $2010-11-14$ & 1200 \\
\hline 55515.10 & $2010-11-15$ & 1200 \\
\hline 55519.10 & 2010-11-19 & 1200 \\
\hline
\end{tabular}

\subsection{Photometric observations at the Wise Observatory}

Mrk 926 was observed with a Bessell V-band filter (Bessell 1990) at the C18 telescope (Brosch et al. 2008) of the Wise Observatory in Israel, from 2010 August 28 to December 10. Observations were carried out daily with gaps due to bad weather on some nights. The SBIG STL-6303 CCD was used with $3072 \times 20489 \mu \mathrm{m}$ pixel, and plate-scale of $1.47 \operatorname{arcsec}_{\mathrm{pix}}{ }^{-1}$, which gives a field of view of $75 \times 50$ arcmin. Each night several exposures of $5 \mathrm{~min}$ each were obtained. The V-band filter has a bandwidth ranging from $4700 \AA$ to $6500 \AA$ and an effective central wavelength of $5415 \AA$. The contribution of the total $\mathrm{H} \beta$ flux to the V band can be estimated to be $<10 \%$. Since the variable part of $\mathrm{H} \beta$ only accounts for $30-40 \%$ of the total $\mathrm{H} \beta$ flux during our campaign (see Table 5) the variable $\mathrm{H} \beta$ contribution to the $\mathrm{V}$ band is $<4 \%$ and is therefore negligible.

Data reduction was carried out in the standard way for bias, dark, and flat-field, using standard IRAF routines. In order to measure the instrumental magnitude of the objects in each image we used the DAOPHOT package (Stetson 1987) as implemented in IRAF. We derived the point-spread function for each image and measured all available objects in the image. We then used the DAOSTAT program (Netzer et al. 1996) to choose the nonvariable stars in the field and use them to inter-calibrate by differential photometry the instrumental magnitude of Mrk 926 between the images and to get its light curve. Measurements from the same night were averaged to obtain a single measurement for each night in order to decrease the uncertainty on each point in the light curve.

\section{Results}

\subsection{Continuum and spectral line variations}

We present all the reduced [O III] $\lambda 5007$-intercalibrated optical spectra of our variability campaign from Mrk 926 in Fig. 2. They clearly show variations in the continuum intensities. The mean and rms spectra of Mrk 926 are shown in Fig. $3{ }^{4}$ The rms spectrum indicates the variable part of the emission lines. It was scaled to allow for a better comparison with the mean spectrum and to enhance weaker line structures. The continuum regions and integration limits of the broad emission lines used in our present study are given in Table 2 and shown at the bottom of the spectra in Fig. 2. We selected the continuum regions by inspecting the mean and rms spectra for regions that are free of both strong emission and absorption lines.

A continuum region at $5100 \AA$ (rest frame) is often used in studies of the variable continuum flux in AGN. Normally, this region is free of strong emission lines and close to the [O III $] \lambda 5007$ flux calibration line. However, in our case this region is contaminated by $\mathrm{H} \beta$ because of the very broad line profile widths in Mrk 926. Thus, we settled for a continuum range slightly more shifted to the red part of the spectrum ( $5180 \AA$ in the rest frame). Finally, we determined the continuum intensities at seven wavelength ranges (at 4425, 4755, 5420, 5485, 5755, 6530 and 7255 $\AA$ in the observed frame). We integrated the broad emission-line intensities of the Balmer and $\mathrm{He}_{\mathrm{I}}$ lines between the wavelength boundaries given in Table 2 . The red wing of the He II $\lambda 4686$ line and the blue wing of $\mathrm{H} \beta$ are superimposed because of their very

\footnotetext{
4 The presented mean and rms spectra are a combination of the blue and red intercalibrations. We merged them at $6345 \AA$ and used a small scaling factor to adapt the redward calibration to the intercalibration with respect to $[\mathrm{O}$ III] $\lambda 5007$.
} 


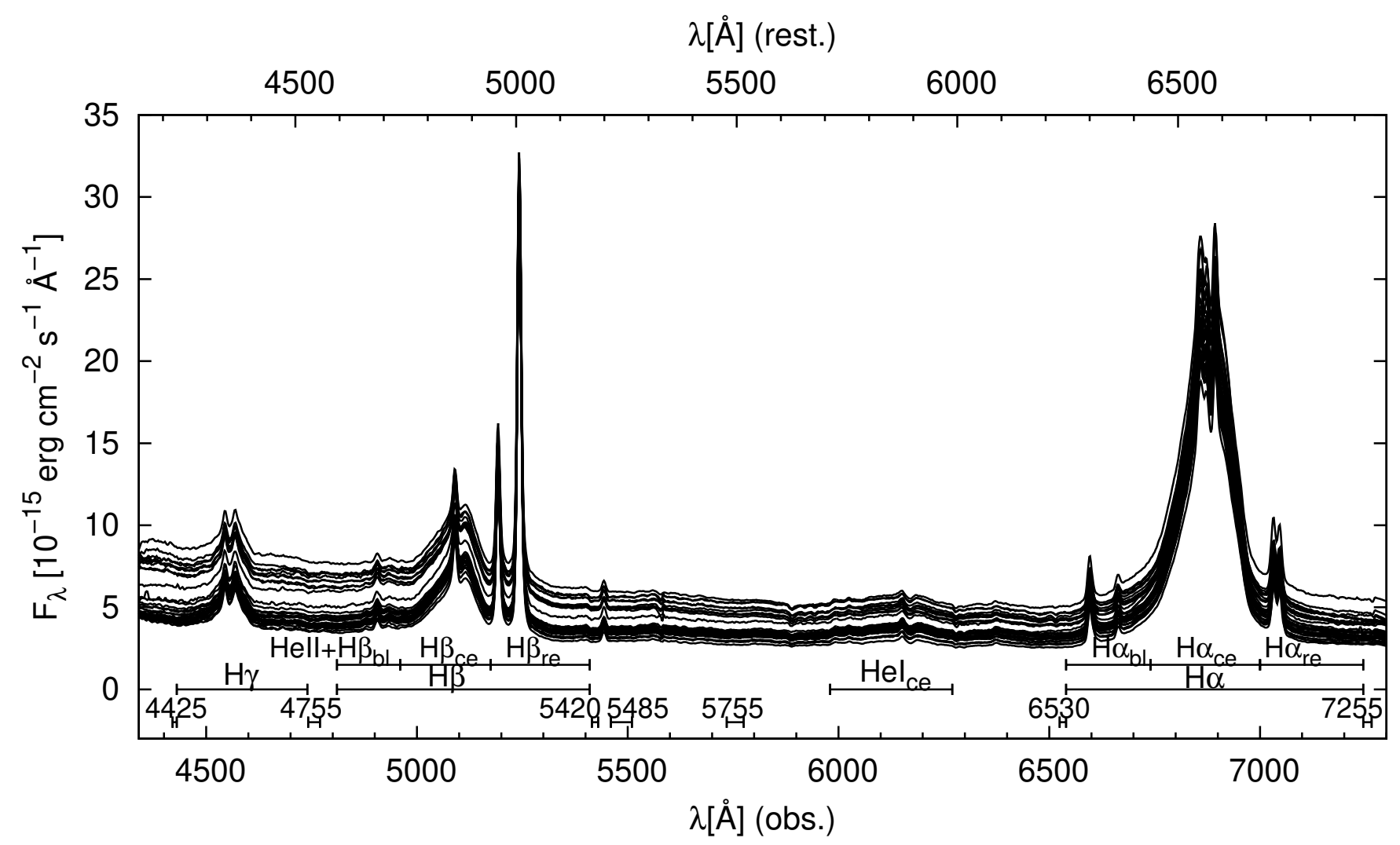

Fig. 2: Reduced and [O III] $\lambda 5007$-intercalibrated optical spectra of Mrk 926 taken with the HET telescope during our variability campaign in 2010. The continuum bands and the broad-line integration intervals are marked according to Table 2 . The spectra shown here are not corrected for Galactic foreground extinction.

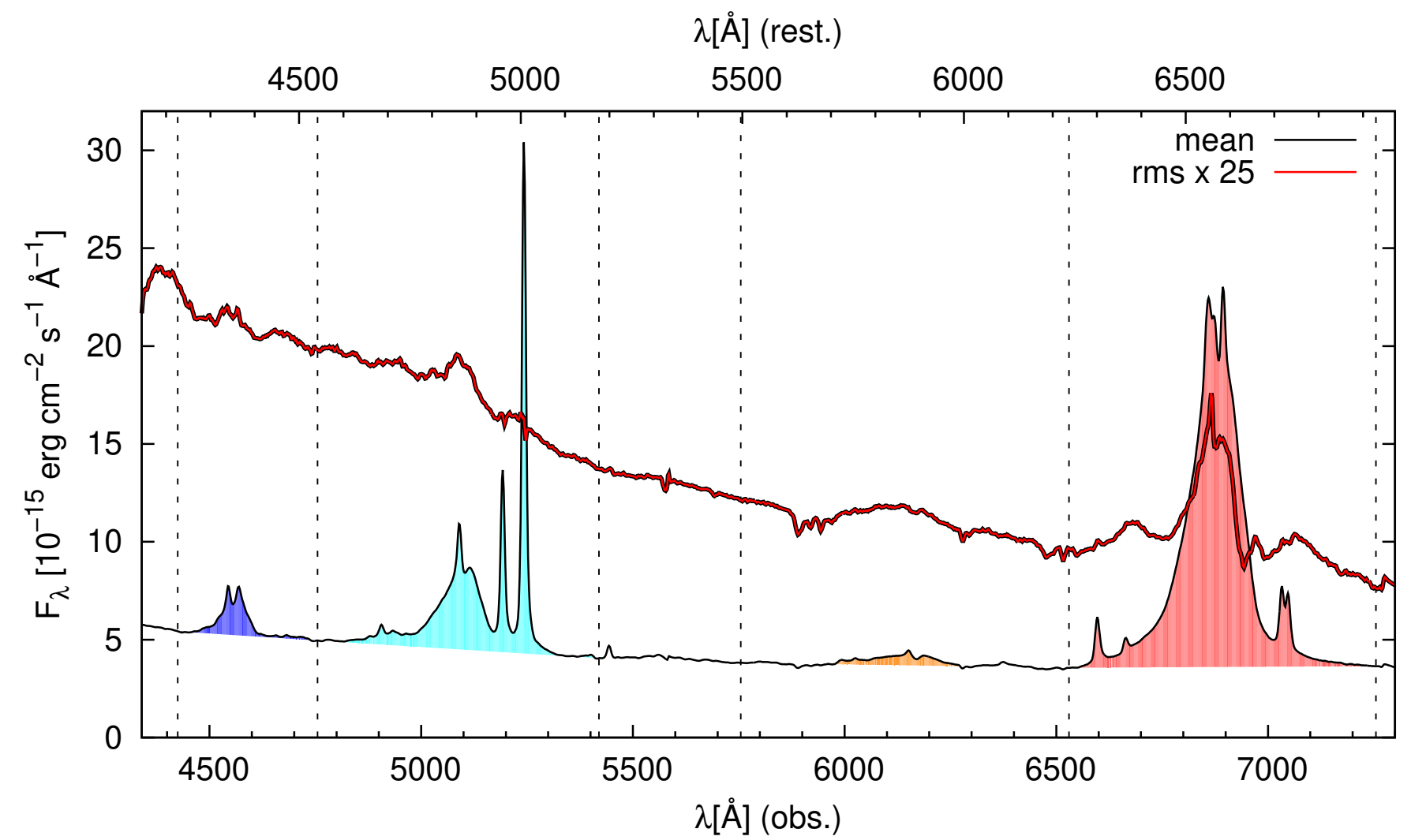

Fig. 3: Combined mean spectrum (black) and rms spectrum (red; see Sect. 3.1 for details) of Mrk 926 from our variability campaign. The rms spectrum was scaled to allow for a direct comparison. The central wavelengths of the pseudo-continua used for linear continuum subtraction are denoted by the vertical dashed lines. The line integration limits are marked by the shaded areas. 
broad line widths. There is no clear minimum between the two lines. Therefore, we assigned the emission-line flux shortward of $4960 \AA$ to a mix of the He II $\lambda 4686$ line and blue $\mathrm{H} \beta$ wing, and the flux longward of $4960 \AA$ to $\mathrm{H} \beta$. Before integrating the emissionline flux, we subtracted a linear pseudo-continuum defined by the continua given in Table 2 (Col. 3 ). The results of the continuum and the integrated line intensity measurements - including the narrow-line components - are given in Tables B.1 and B.2. The light curves of the integrated emission-line fluxes of $\mathrm{H} \alpha, \mathrm{H} \beta$, $\mathrm{H} \gamma, \mathrm{H} \beta_{\text {blue }}+\mathrm{He}$ II $\lambda 4686$, and $\mathrm{He}$ I $\lambda 5876_{\text {center }}$ are given in Fig. 6. For further analysis, we also measured the narrow-line components of each broad line in the mean spectrum. The de-blending process of the narrow and broad components in the Balmer and He $\mathrm{I} \lambda 5876$ lines is described in more detail in Sect. 3.4. The resulting narrow-line fluxes are presented in Table 3.

\subsubsection{Intercalibration of HET and Wise light curves}

We present the light curves of the continuum flux densities at $4425,4755,5420,5485,5744$, and $6530 \AA$ (observed frame) in Fig. 4. In order to increase the temporal resolution, we created a combined continuum light curve based on the HET continuum flux densities at $5420 \AA$ and on the photometric V-band continuum light curve obtained at the Wise Observatory. We followed the procedure described in Grier et al. (2012) and Zetzl et al. (2018) by applying a multiplicative scale factor and an additional flux adjustment component to put the Wise Observatory instrumental magnitude light curve on the same scale as the HET continuum light curve in order to correct for different instrumentations, differences in the aperture sizes and therefore different host galaxy contributions, and constant contributions from emission lines within the bandwidth of the V-band filter. The optimal scale factor and flux adjustment component were determined by finding the set of parameters for which the cumulative difference of (nearly) simultane-

Table 2: Continuum boundaries, line integration limits, and pseudo-continua.

\begin{tabular}{lcc}
\hline \hline $\begin{array}{l}\text { Cont./Line } \\
(1)\end{array}$ & $\begin{array}{c}\text { Wavelength Range } \\
(2)\end{array}$ & $\begin{array}{c}\text { Pseudo-Continuum } \\
(3)\end{array}$ \\
\hline Cont. 4425 (4225) & $4420-4430$ & \\
Cont. 4755 (4540) & $4742-4770$ & \\
Cont. 5420 (5180) & $5415-5430$ & \\
Cont. 5485 (5240) & $5460-5510$ & \\
Cont. 5755 (5500) & $5735-5775$ & \\
Cont. 6530 (6240) & $6525-6540$ & \\
Cont. 7255 (6930) & $7245-7265$ & \\
\hline $\mathrm{H} \gamma$ & $4430-4740$ & $4425-4755$ \\
$\mathrm{H} \beta$ & $4810-5410$ & $4755-5420$ \\
$\mathrm{H} \beta_{\text {blue }}+\mathrm{He}$ II $\lambda 4686$ & $4810-4960$ & $4755-5420$ \\
$\mathrm{H} \beta_{\text {center }}$ & $4960-5175$ & $4755-5420$ \\
$\mathrm{H} \beta_{\text {red }}$ & $5175-5410$ & $4755-5420$ \\
$\mathrm{He}$ I $\lambda 5876_{\text {center }}$ & $5980-6270$ & $5755-6530$ \\
$\mathrm{H} \alpha$ & $6540-7245$ & $6530-7255$ \\
$\mathrm{H} \alpha_{\text {blue }}$ & $6540-6740$ & $6530-7255$ \\
$\mathrm{H} \alpha_{\text {center }}$ & $6740-7000$ & $6530-7255$ \\
$\mathrm{H} \alpha_{\text {red }}$ & $7000-7245$ & $6530-7255$ \\
\hline
\end{tabular}

Notes. The continuum wavelength values in brackets correspond to the rest frame of Mrk 926. All wavelengths are given in $\AA$.
Table 3: Observed narrow-emission-line fluxes as measured in the mean spectrum shown in Fig. 3.

\begin{tabular}{lr|lr}
\hline \hline Line & \multicolumn{1}{c}{ Flux } & Line & \multicolumn{1}{c}{ Flux } \\
\hline $\mathrm{H} \gamma_{\text {narrow }}$ & $7.4 \pm 1.0$ & [O I $] \lambda 6363$ & $8.0 \pm 1.5$ \\
$\mathrm{He}$ II $\lambda 4686$ & $6.3 \pm 0.9$ & {$[\mathrm{~N}$ II] $\lambda 6548$} & $18.8 \pm 3.0$ \\
$\mathrm{H} \beta_{\text {narrow }}$ & $15.7 \pm 1.8$ & $\mathrm{H} \alpha_{\text {narrow }}$ & $42.4 \pm 3.4$ \\
{$\left[\mathrm{O}_{\text {III }}\right] \lambda 4959$} & $102 . \pm 2.1$ & {$\left[\mathrm{~N}_{\mathrm{II}}\right] \lambda 6584$} & $54.0 \pm 3.4$ \\
{$\left[\mathrm{O}_{\text {III }}\right] \lambda 5007$} & $314 . \pm 3.3$ & {$\left[\mathrm{~S}_{\mathrm{II}}\right] \lambda 6717$} & $41.1 \pm 2.1$ \\
$\mathrm{He}_{\mathrm{I}} \lambda 5876$ & $3.1 \pm 0.7$ & {$[\mathrm{~S}$ II] $\lambda 6731$} & $37.0 \pm 2.0$ \\
{$\left[\mathrm{O}_{\mathrm{I}}\right] \lambda 6300$} & $33.8 \pm 2.7$ & & \\
\hline
\end{tabular}

Notes. In units of $10^{-15} \mathrm{erg} \mathrm{cm}^{-2} \mathrm{~s}^{-1}$.

ous spectroscopic and photometric continuum measurements reached its minimum. The resulting combined light curve is given in Table B.4 and shown in Fig. 5. Excluding the first two data points, the time interval between observations amounts to only 1.1 days. For further analysis, we corrected this light curve for Galactic foreground extinction $\left(A_{\mathrm{V}}=0.113\right.$; Schlafly $\&$ Finkbeiner 2011) and subtracted the V-band host galaxy contribution $\left(1.35 \times 10^{-15} \mathrm{erg} \mathrm{cm}^{-2} \mathrm{~s}^{-1} \AA^{-1}\right.$; see Sect. 3.2). For comparison, we also show the combined continuum light curve in Figs. 6, A.1, and A.2.

\subsection{Host galaxy contribution to the optical continuum flux}

The observed flux of the variable AGN component is contaminated by the flux contribution of the host galaxy. This contribution is nearly constant for observations taken with identical aperture, and only minor deviations arise due to slight variations from the exact pointing as well as different seeing conditions. However, these effects are taken into account by our error estimates. The overall host galaxy contribution varies from band to band as the stellar component of the host galaxy has a different flux distribution than the AGN component. To estimate the relative contribution of the host galaxy flux, we used the flux variation gradient (FVG) method (Choloniewski 1981; Winkler et al. 1992; Haas et al. 2011; Zetzl et al. 2018). This method allows the varying AGN flux in our aperture to be disentangled from the host galaxy contribution. We took the continuum flux densities at 4425,5420 , and $6530 \AA$ (observed frame) as proxies for B-, V-, and R-band fluxes, respectively, since these continuum ranges are close to the maxima of the $\mathrm{B}-, \mathrm{V}-$, and R-filter curves (B-band filter: $4300 \AA$, V-band filter: $5400 \AA$ A, R-band filter: 6100 $\AA$ ). In this way, we also excluded the contribution of emission lines (see Fig. 2). Figure 7 shows the B versus V and B versus R fluxes (black solid circles) of Mrk 926 based on the HET spectra (aperture: $2 \times 2$ arcsec). The $\mathrm{B}, \mathrm{V}$, and $\mathrm{R}$ values in this figure have been corrected for Galactic foreground extinction $\left(\mathrm{A}_{B}=\right.$ $0.149, \mathrm{~A}_{V}=0.113, \mathrm{~A}_{R}=0.089$; Schlafly \& Finkbeiner (2011)) and are given in units of $\mathrm{mJy}$. The converted light curves before and after Galactic foreground extinction are given in Table B.3. The blue dashed line in Fig. 7 gives the best linear fit to the B versus $\mathrm{V}$ and $\mathrm{B}$ versus $\mathrm{R}$ fluxes. The red shaded area marks the range of host slopes for nearby AGN as determined by Sakata et al. (2010). The intersection point between the AGN and host galaxy slopes allows the contribution of the host galaxy fluxes in the $\mathrm{B}, \mathrm{V}$, and $\mathrm{R}$ bands to be determined; the resulting host galaxy contributions in the individual bands are indicated by the dashed black lines. 
A\&A proofs: manuscript no. MRK926_220330_arxiv_v2

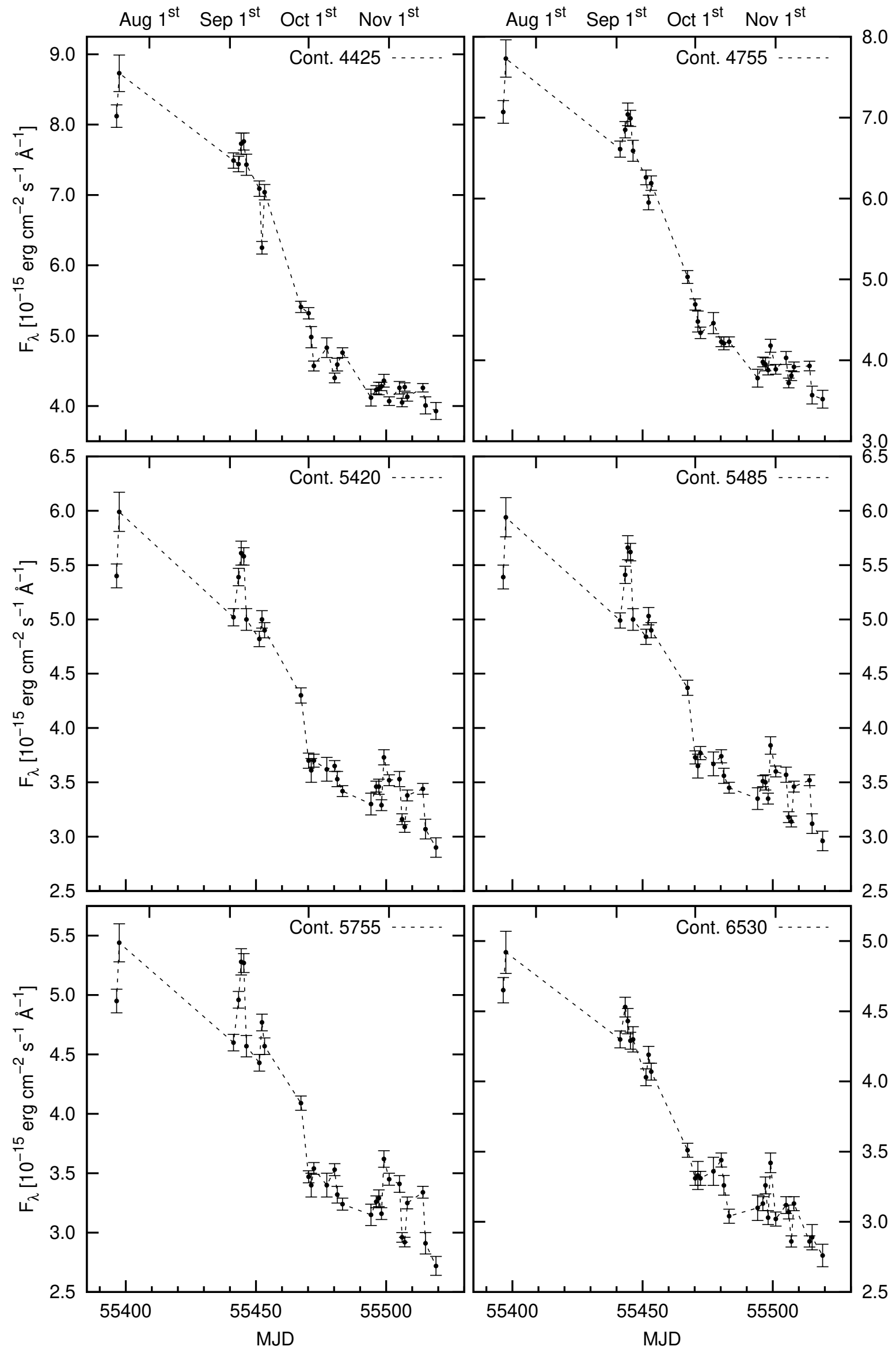

Fig. 4: Light curves of the continuum flux densities at 4425, 4755, 5420, 5485, 5755, and $6530 \AA$ (observed frame; in units of $10^{-15}$ $\operatorname{erg~cm} \mathrm{cm}^{-2} \mathrm{~s}^{-1} \AA^{-1}$ ) of our HET variability campaign from 2010 July 19 to November 19 . 


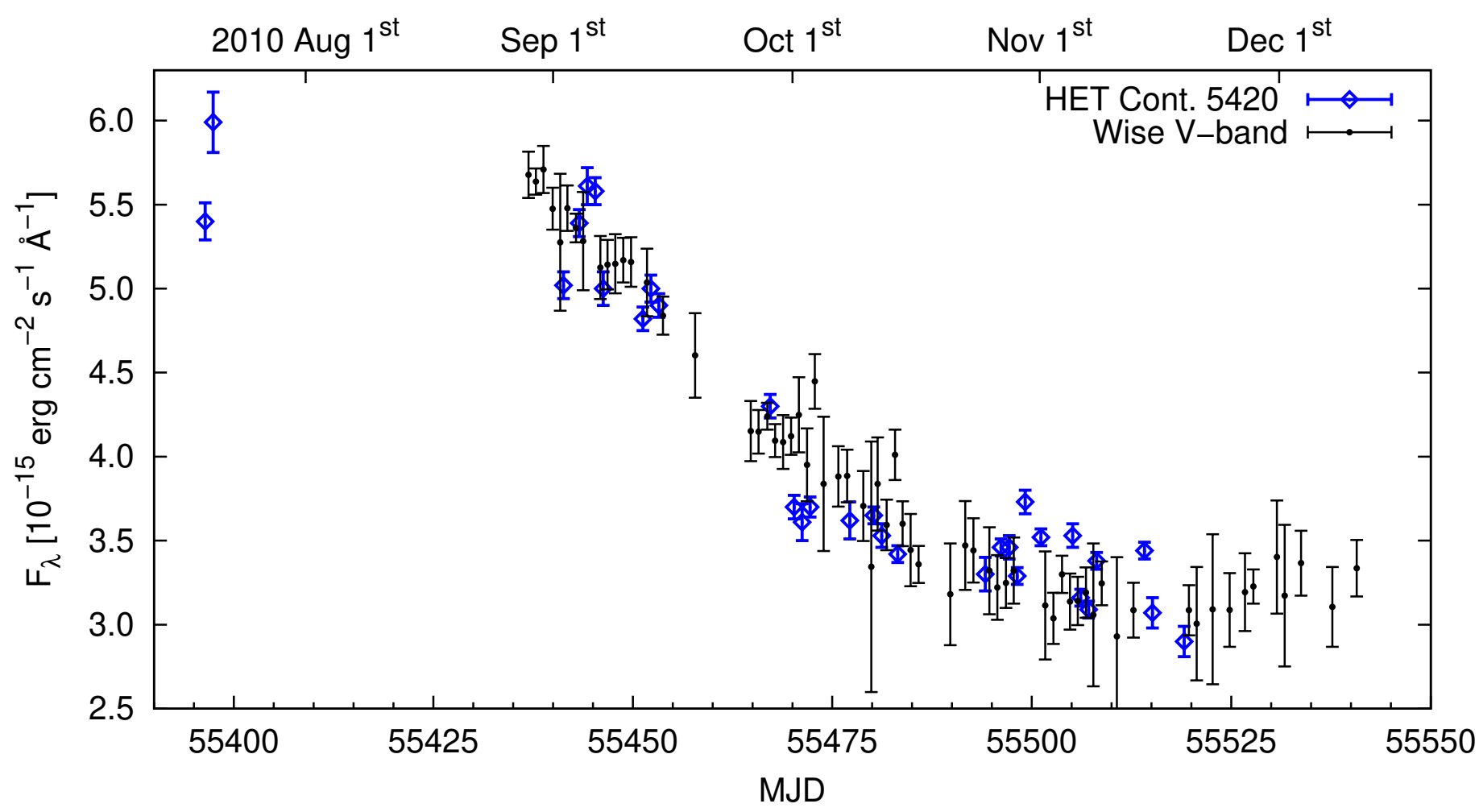

Fig. 5: Combined spectroscopic (HET continuum flux densities at $5420 \AA$, or $5180 \AA$ in rest frame; blue) and photometric (V-band continuum data obtained at the Wise Observatory; black) continuum light curve of our campaign (see Sect. 3.1 for details).

We derived a B-band host galaxy contribution of $0.76 \mathrm{mJy}$ (mean of $0.56 \mathrm{mJy}$ and $0.96 \mathrm{mJy}$ ). The corresponding values are $1.32 \mathrm{mJy}$ for the $\mathrm{V}$ band and $2.82 \mathrm{mJy}$ for the R band. By subtracting the host galaxy contribution for each individual band, we isolate the AGN flux in all bands. All flux values (minimal and maximal host+AGN, host contribution as well as true minimal and maximal AGN flux) are listed in Table 4. Based on the HET spectra, the host galaxy contribution corresponds to 12 $26 \%, 20-42 \%$, and $37-66 \%$ for the B, V, and R bands, respectively. Altogether, the spectra of Mrk 926 are dominated by emission from the nucleus. The weak host contribution in the B and $\mathrm{V}$ bands and, especially, the very strong and broad emission lines prevent us from subtracting a reliable host galaxy template since the stellar signature of the host galaxy is suppressed.

\subsection{Variability statistics}

The following statistics are based on the spectroscopic and photometric observations from HET and the Wise Observatory, respectively. The continua were corrected for Galactic foreground extinction (see Sect. 3.1), and the lines were corrected for contribution from the narrow lines (see Table 3 ). In Table 5, we present the minimum and maximum fluxes, $F_{\min }$ and $F_{\max }$, peak-to-peak amplitudes, $R_{\max }=F_{\max } / F_{\min }$, the mean flux over the period of observations, $\langle F\rangle$, the standard deviation, $\sigma_{F}$, and the fractional variation,

$F_{\mathrm{var}}=\sqrt{\sigma_{F}^{2}-\Delta^{2}} /<F>$,

as defined by Rodríguez-Pascual et al. (1997). The quantity $\Delta^{2}$ is the mean square value of the uncertainties $\Delta_{\mathrm{i}}$ associated with the fluxes $F_{\mathrm{i}}$. The $F_{\text {var }}$ uncertainties are defined in Edelson et al. (2002). The peak-to-peak amplitudes and the fractional variations in the continuum decrease as a function of wavelength.
Table 4: Extinction-corrected B, V, and R values for the combined host galaxy plus AGN fluxes as well as for the host galaxy and AGN fluxes alone as determined by the FVG method. When a range is given, it corresponds to the minimum and maximum flux.

\begin{tabular}{|c|c|c|c|}
\hline Flux Component & $\mathrm{B}$ band & $\mathrm{V}$ band & $\mathrm{R}$ band \\
\hline$(1)$ & $(2)$ & $\begin{array}{c}{[\mathrm{mJy}]} \\
(3)\end{array}$ & (4) \\
\hline $\begin{array}{l}\text { Host+AGN (BvsR) } \\
\text { Host+AGN (BvsV) }\end{array}$ & $\begin{array}{l}2.94-6.54 \\
2.94-6.54\end{array}$ & $3.15-6.51$ & $4.26-7.60$ \\
\hline $\begin{array}{l}\text { Host (BvsR) } \\
\text { Host (BvsV) }\end{array}$ & $\begin{array}{l}0.96 \\
0.56\end{array}$ & 1.32 & 2.82 \\
\hline $\begin{array}{l}\text { AGN (BvsR) } \\
\text { AGN (BvsV) }\end{array}$ & $\begin{array}{l}1.98-5.58 \\
2.39-5.98 \\
\end{array}$ & $1.83-5.19$ & $1.44-4.78$ \\
\hline (1) & $(2)^{\left[10^{-1}\right.}$ & $\begin{array}{c}\operatorname{erg~s}^{-1} \mathrm{~cm}^{-2} \\
\text { (3) }\end{array}$ & $\left.\AA^{-1}\right]$ (4) \\
\hline $\begin{array}{l}\text { Host+AGN (BvsR) } \\
\text { Host+AGN (BvsV) }\end{array}$ & $\begin{array}{l}4.51-10.01 \\
4.51-10.01\end{array}$ & $3.22-6.65$ & $3.00-5.34$ \\
\hline $\begin{array}{l}\text { Host (BvsR) } \\
\text { Host (BvsV) }\end{array}$ & $\begin{array}{l}1.47 \\
0.86\end{array}$ & 1.35 & 1.98 \\
\hline $\begin{array}{l}\text { AGN (BvsR) } \\
\text { AGN (BvsV) }\end{array}$ & $\begin{array}{l}3.03-8.54 \\
3.66-9.16\end{array}$ & $1.87-5.30$ & $1.01-3.36$ \\
\hline
\end{tabular}

\subsection{Mean and rms line profiles}

We determined normalized mean and rms profiles of the Balmer and He $\mathrm{I}$ lines in Mrk926 after subtracting a linear pseudocontinuum from each mean and rms broad-line profile. In order to obtain the true mean broad-line profiles, we subtracted 
A\&A proofs: manuscript no. MRK926_220330_arxiv_v2

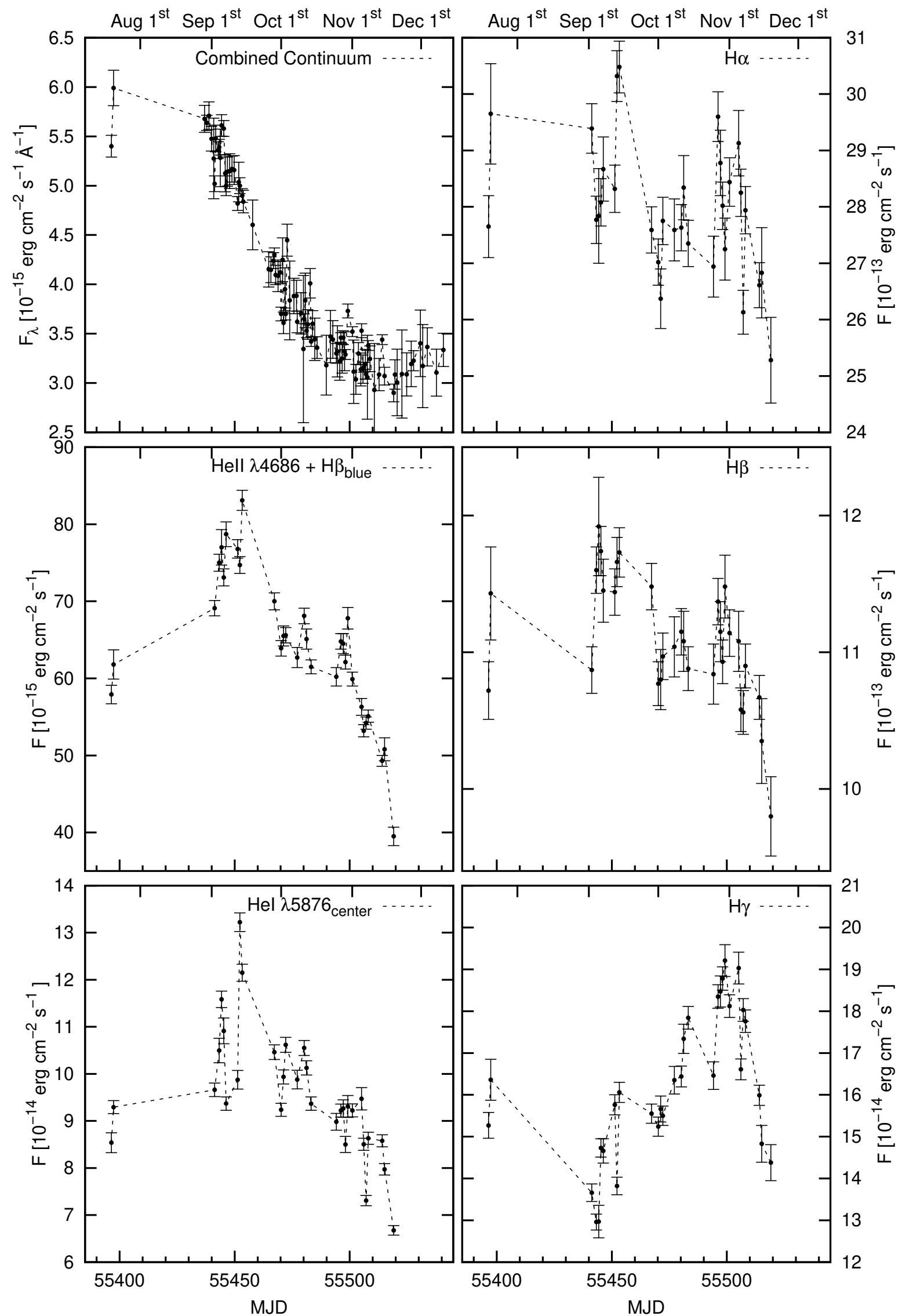

Fig. 6: Light curves of the combined continuum flux densities at $5420 \AA$ (observed frame; in units of $10^{-15} \mathrm{erg} \mathrm{cm}^{-2} \mathrm{~s}^{-1} \AA^{-1}$ ) as well as of the integrated emission-line fluxes of $\mathrm{H} \alpha, \mathrm{H} \beta, \mathrm{H} \gamma, \mathrm{He}$ II $\lambda 4686+\mathrm{H} \beta_{\text {blue }}$, and $\mathrm{He}$ I $\lambda 5876$ for our HET variability campaign from 2010 July 19 to November 19. 

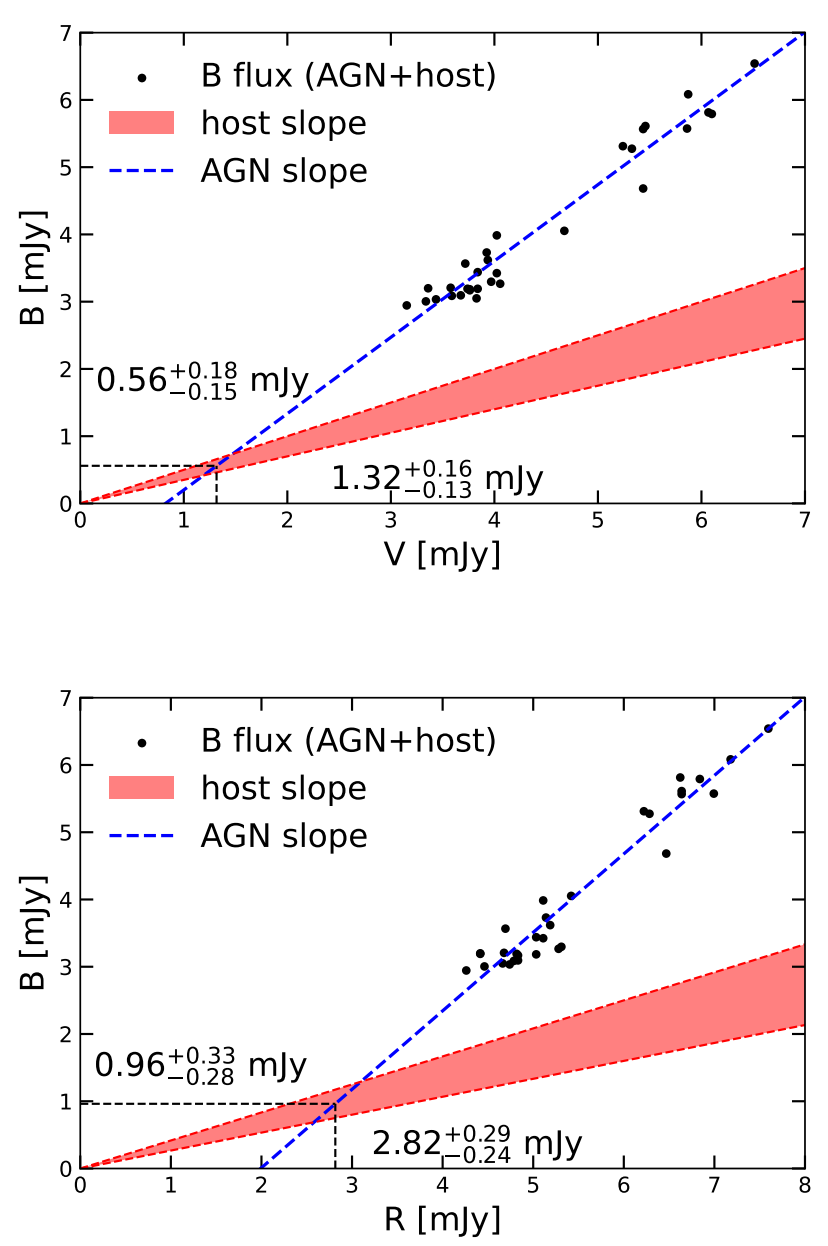

Fig. 7: Extinction-corrected B versus V (upper panel) and B versus R (lower panel) flux variations of Mrk 926. The dashed blue lines (AGN slope) represent the best linear fit to the B versus V and $B$ versus $R$ fluxes, respectively. The red shaded area shows the range of host slopes. The dashed black lines indicate the $\mathrm{B}$ and $\mathrm{V}$ as well as the $\mathrm{B}$ and $\mathrm{R}$ values of the host galaxy.

the narrow Balmer and $\mathrm{He}$ I line components in velocity-space by means of a scaled [O III] $\lambda 5007$ template based on the observed $\left[\mathrm{O}_{\mathrm{III}}\right] \lambda 5007$ line profile in our mean spectrum. Furthermore, we subtracted the [NII]6548/6584 emission lines that are superimposed on the mean $\mathrm{H} \alpha$ profile using the same template and sticking to a line-ratio of 1:3. The rms profiles illustrate the line profile variations during our campaign. Therefore, the constant narrow components disappear in these rms profiles. Finally, we normalized both the mean and rms profiles such that the maximum line flux was set to unity. The final normalized mean and rms profiles of the Balmer lines $\mathrm{H} \alpha, \mathrm{H} \beta, \mathrm{H} \gamma$, as well as of the He $\mathrm{r} \lambda 5876$ line in velocity space are shown in Fig. 8. The subtraction of all narrow Balmer and He I components as well as of [NII]6548/6584 and the normalization of the true broad-line profiles allows the mean and rms profiles to be compared with each other in a more accurate way.

The Balmer lines exhibit - besides to their central component - an additional inner red component at $1200 \pm 300 \mathrm{~km} \mathrm{~s}^{-1}$ in the mean as well as rms profiles (see Fig. 8). Furthermore, the Balmer lines show additional outer broad-line components
Table 5: Variability statistics $\left(F_{\min }, F_{\max }, R_{\max },<F>, \sigma_{\mathrm{F}}\right.$, and $\mathrm{F}_{\mathrm{var}}$ ) for the continuum (after correction for Galactic foreground extinction) and for the broad emission lines (after correction for the contribution of narrow-line components; see Table 3).

\begin{tabular}{lrrrrrr}
\hline \hline Cont./Line & $F_{\min }$ & $F_{\max }$ & $R_{\max }$ & $\langle F\rangle$ & $\sigma_{\mathrm{F}}$ & $F_{\text {var }}$ \\
\hline Comb. V band & 3.22 & 6.65 & 2.07 & 4.41 & 0.97 & 0.21 \\
\hline Cont. 4425 (4225) & 4.51 & 10.01 & 2.22 & 6.22 & 1.77 & 0.28 \\
Cont. 4755 (4540) & 4.04 & 8.87 & 2.20 & 5.67 & 1.52 & 0.27 \\
Cont. 5420 (5180) & 3.22 & 6.65 & 2.07 & 4.50 & 1.01 & 0.22 \\
Cont. 5485 (5240) & 3.28 & 6.59 & 2.01 & 4.54 & 0.99 & 0.22 \\
Cont. 5755 (5500) & 3.02 & 6.04 & 2.00 & 4.23 & 0.90 & 0.21 \\
Cont. 6530 (6240) & 3.00 & 5.34 & 1.78 & 3.85 & 0.68 & 0.17 \\
Cont. 7255 (6930) & 3.20 & 5.03 & 1.57 & 3.93 & 0.54 & 0.14 \\
\hline $\mathrm{H} \gamma$ & 122.2 & 184.7 & 1.51 & 154.6 & 17.6 & 0.11 \\
$\mathrm{H} \beta$ & 542. & 754. & 1.39 & 670. & 46. & 0.07 \\
$\mathrm{H} \beta_{\text {blue }}+\mathrm{He}$ II $\lambda 4686$ & 33.2 & 76.80 & 2.31 & 57.8 & 9.6 & 0.17 \\
$\mathrm{H} \beta_{\text {center }}$ & 419. & 556. & 1.33 & 502. & 30. & 0.06 \\
$\mathrm{H} \beta_{\text {red }}$ & 89.2 & 126.8 & 1.42 & 109.5 & 8.5 & 0.08 \\
$\mathrm{HeI} \lambda 5876_{\text {center }}$ & 63.7 & 129.1 & 2.03 & 92.7 & 13.2 & 0.14 \\
$\mathrm{H} \alpha$ & 2293. & 2813. & 1.23 & 2562. & 118. & 0.04 \\
$\mathrm{H} \alpha_{\text {blue }}$ & 131.3 & 192.4 & 1.47 & 159.2 & 14.7 & 0.09 \\
$\mathrm{H} \alpha_{\text {center }}$ & 2082. & 2456. & 1.18 & 2286. & 93.3 & 0.04 \\
$\mathrm{H} \alpha_{\text {red }}$ & 79.6 & 166.9 & 2.10 & 116.1 & 23.9 & 0.21 \\
\hline
\end{tabular}

Notes. In units of $10^{-15} \mathrm{erg} \mathrm{s}^{-1} \mathrm{~cm}^{-2} \AA^{-1}$ for the continuum and in units of $10^{-15} \mathrm{erg} \mathrm{s}^{-1} \mathrm{~cm}^{-2}$ for the broad emission lines. The fluxes in this table are observed-frame fluxes. The continuum wavelength values in brackets correspond to the rest frame of Mrk 926.

in their rms profiles (Fig. 8) at \pm 5000 to $\pm 13000 \mathrm{~km} \mathrm{~s}^{-1}$. These Balmer satellites are clearly separated from the central component $\left( \pm 5000 \mathrm{~km} \mathrm{~s}^{-1}\right)$ and exhibit an amplitude that amounts to $25-40 \%$ of that of the maximum rms amplitude in $\mathrm{H} \alpha$ and $\mathrm{H} \beta$, respectively. These components are not recognizable in the mean profiles. The $\mathrm{H} \gamma$ profile is contaminated by the [OIII] $\lambda 4363$ line and a strong residual is present in the rms profile. This is due to $\mathrm{H} \gamma$ being at the blue end of the spectrum where the flux calibration for the first few pixels is not optimal. Furthermore, it is not possible to fix a line-free continuum region on the blue side. These boundary effects heavily affect the blue part of the $\mathrm{H} \gamma$ line profile. Therefore, the $\mathrm{H} \gamma$ profile can only be used to derive some general trends. Despite the boundary effects mentioned before, the rms profile of $\mathrm{H} \gamma$ confirms the presence of Balmer satellites at \pm 5000 to $\pm 13000 \mathrm{~km} \mathrm{~s}^{-1}$. The red wing of He I $\lambda 5876 \mathrm{might}$ also indicate the presence of such a line satellite, however, a clear detection cannot be stated due to the interference by absorption in the line wings. In addition, the central He $\mathrm{I} \lambda 5876$ profile is contaminated by $\mathrm{NaD}$ absorption.

We determined the line widths (FWHM) of the mean and rms line profiles of all the Balmer lines and He i 25876 . Additionally, we parameterized the line widths of the mean profiles by their line dispersion $\sigma_{\text {line }}$ (Fromerth \& Melia 2000; Peterson et al. 2004). However, due to the very broad, partially overlapping and complex rms line profiles with additional outer components, it was not possible do determine reliable $\sigma_{\text {line }}$ (rms) values. Likewise, by cause of $\mathrm{H} \gamma$ being affected by boundary effects, the overlapping $\mathrm{He}$ II $\lambda 4686$ and $\mathrm{H} \beta_{\text {blue }}$ profiles, as well as strong absorption in $\mathrm{He}_{\mathrm{I}} \lambda 5876$, a determination of $\sigma_{\text {line }}$ (mean) for these lines was deemed unreliable. Therefore, we determined 

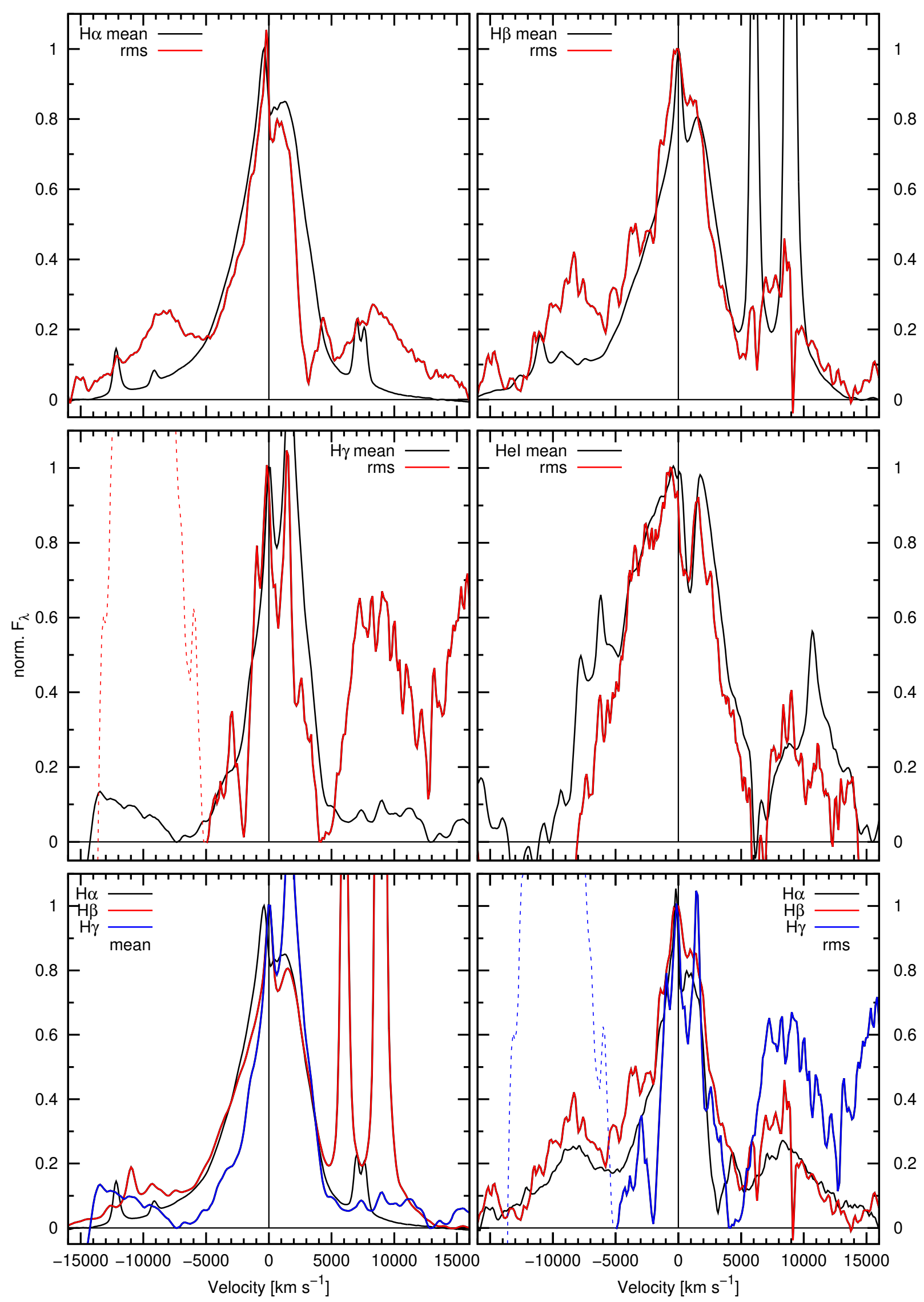

Fig. 8: Normalized mean and rms profiles of the Balmer lines and HeI $\lambda 5876$ in velocity space. The dashed part of the $\mathrm{H} \gamma$ line profile denotes the part that is heavily affected by uncertainties in the flux calibration due to boundary effects at the blue end of the spectrum.

Article number, page 10 of 23 
the line dispersion $\sigma_{\text {line }}$ (mean) solely for the $\mathrm{H} \alpha$ mean profile over a velocity interval from -15000 to $+15000 \mathrm{~km} \mathrm{~s}^{-1}$.

The resulting widths (FWHM) for all emission lines, and the line dispersion of $\mathrm{H} \alpha\left(\sim 3500 \mathrm{~km} \mathrm{~s}^{-1}\right)$ are given in Table 6 . The $\mathrm{H} \alpha$ and $\mathrm{H} \beta$ lines show similar mean profiles with line widths (FWHM) of $\sim 5200 \mathrm{~km} \mathrm{~s}^{-1}$ as well as similar rms profiles with line widths (FWHM) of $\sim 4000 \mathrm{~km} \mathrm{~s}^{-1}$. The $\mathrm{H} \gamma$ line exhibits a profile similar to that of $\mathrm{H} \alpha$ and $\mathrm{H} \beta$, but the line width and line dispersion are smaller than for the other Balmer lines. We note, however, that these results are less reliable due to the aforementioned boundary effects. The He r $\lambda 5876$ line (mean and rms) is by a factor of about 1.5 broader than the Balmer lines.

Table 6: FWHM of the mean and rms line profiles as well as the line dispersion, $\sigma_{\text {line }}$, of the mean profiles.

\begin{tabular}{lccc}
\hline \hline Line & $\begin{array}{c}\text { FWHM (mean) } \\
{\left[\mathrm{km} \mathrm{s}^{-1}\right]}\end{array}$ & $\begin{array}{c}\text { FWHM (rms) } \\
{\left[\mathrm{km} \mathrm{s}^{-1}\right]}\end{array}$ & $\begin{array}{c}\sigma_{\text {line }}(\text { mean }) \\
{\left[\mathrm{km} \mathrm{s}^{-1}\right]}\end{array}$ \\
\hline $\mathrm{H} \alpha$ & $5370 \pm 400$ & $3890 \pm 500$ & $3460 \pm 1000$ \\
$\mathrm{H} \beta$ & $5110 \pm 400$ & $4230 \pm 400$ & - \\
$\mathrm{H} \gamma$ & $4300 \pm 1000$ & $3260 \pm 800$ & - \\
$\mathrm{He}$ I $\lambda 5876$ & $8500 \pm 1000$ & $7650 \pm 800$ & - \\
\hline
\end{tabular}

\subsection{CCF analysis of the broad emission lines}

The mean distances of the broad emission-line regions to the central ionizing source can be determined by correlating the broad emission-line light curves with the light curve of the ionizing continuum flux. Normally, an optical continuum light curve is used as surrogate for the ionizing light curve. For this study, we correlated the integrated light curves as well as the segment light curves (center, blue, red) of the Balmer lines $\mathrm{H} \alpha$ and $\mathrm{H} \beta$, and the $\mathrm{He}_{\mathrm{I}} \lambda 5876_{\text {center }}$ line with the combined $5180 \AA$ (rest frame) and V-band continuum light curve (see Fig. 5 in Sect. 3.1). For $\mathrm{H} \gamma$, the boundary effects mentioned in Sect. 3.4 prevented a reliable light curve extraction, and therefore no cross-correlation function $(\mathrm{CCF})$ was calculated. The correlation technique we used is a variant of the discrete correlation function (DCF; Edelson \& Krolik 1988). Instead of classically binning the data points (i.e., using a rectangular weighting function), we performed a weighted averaging of the data points using a Gaussian kernel $b_{\tau}(t)=(1 / \sqrt{2 \pi h}) \exp \left(-(\tau-t)^{2} / 2 h^{2}\right)$ as a smooth density function (Rehfeld et al. 2011, and references therein). The width $h$ was chosen such that it was on the order of the mean sampling rate, which in our case translates to $\sim 2$ days. The Pearson correlation coefficient, $\rho_{x, y}=\operatorname{COV}(x, y) /\left(\sigma_{x} \sigma_{y}\right)$, of two time series, $x$ and $y$, with time steps $t^{x}$ and $t^{y}$ then transforms to

$$
\operatorname{CCF}(\tau)=\frac{\sum_{i=1}^{N_{x}}\left(x_{i}-\mu_{x}\right) \cdot\left(z_{i}^{\tau}-\mu_{z^{\tau}}\right)}{N_{x} \sigma_{x} \sigma_{z}},
$$

with $z_{i}^{\tau}=\sum_{j=1}^{N_{y}} y_{j} \cdot b_{\tau}\left(t_{j}^{y}-t_{i}^{x}\right)$. The normalization of the CCF ensures that $\operatorname{CCF}(\tau) \leq 1$ for all $\tau$. We note that the light curves were not detrended as the timescale of the campaign does not suffice to remove long-term trends. The derived cross-correlation functions, $\operatorname{CCF}(\tau)$, are shown in Fig. 9.

We determined the centroids $\tau_{\text {cent }}$ of the CCFs by using only those parts of the CCFs above $80 \%$ of the peak value. A threshold value of $0.8 r_{\max }$ is generally a good choice as has been shown before (Peterson et al. 2004). We derive the uncertainties of the time lags, $\tau_{\text {cent }}$, by calculating the cross-correlation lags a large number of times using a model-independent Monte Carlo method known as flux randomization/random subset selection (FR/RSS). This method has been described by Peterson et al. (1998). The resulting cross-correlation centroid distributions (CCCDs) for the integrated $\mathrm{H} \alpha, \mathrm{H} \beta$, and $\mathrm{He}$ I lines as well as for their line segments (center, blue, red) with the combined $5180 \AA$ (rest frame) and V-band continuum are presented in Fig. 9. Each CCCD was determined after $2 \times 10^{4}$ independent runs (i.e., $2 \times 10^{4}$ independent subsamples) for each light curve. For the CCFs of the central Balmer line segments showing two distinct peaks, the CCCD was calculated for each peak individually. The final centroid time lags are given in Table 7 . The error intervals correspond to $68 \%( \pm 1 \sigma)$ confidence level.

The resulting CCFs and CCCDs are presented in Fig. 9. The $\mathrm{CCFs}$ of the integrated Balmer line light curves and of the central Balmer line component light curves (within $\pm 5000 \mathrm{~km} \mathrm{~s}^{-1}$ ) exhibit two discrete peaks. In contrast, the blue and red Balmer segment light curves (i.e., the light curves of the Balmer satellites) are single-peaked. The double-peaked CCFs of the integrated Balmer line light curves show delays of $8_{-5}^{+2}$ and $56_{-6}^{+3}$ days for $\mathrm{H} \alpha$, and $5_{-2}^{+2}$ and $48_{-6}^{+9}$ days for $\mathrm{H} \beta$. The CCFs of the central Balmer line component light curves show delays of $10_{-2}^{+3}$ and $57_{-3}^{+3}$ days for $\mathrm{H} \alpha$, and $5_{-2}^{+2}$ and $48_{-6}^{+7}$ days for $\mathrm{H} \beta$. The interpretation of these double-peaked CCFs is explained in more detail in Sect. 3.6. The CCFs of the Balmer satellite light curves are single-peaked and show, in comparison to the CCFs of the integrated and central component light curves, much shorter delays of only 3-5 light-days (i.e., the Balmer satellites react promptly to the variations in the continuum). There are indications for a secondary peak in the red Balmer satellite CCF of $\mathrm{H} \beta$ (see Fig. 9c); however, the light curve of this wing is relatively noisy due to the strong underlying [O III] lines (see Sect. 4.5.2 for more information). The CCF of the blue $\mathrm{H} \beta$ Balmer satellite shows no indication of a secondary peak.

We also calculated the CCFs and CCCDs for the weak HeI $\lambda 5876$ line. The outer wings are heavily contaminated by absorption. Therefore, out of precaution we excluded the blue and red wing from the integrated line light curve, leaving only the central profile light curve. In contrast to the Balmer lines, the $\mathrm{CCF}$ of the central profile light curve only shows a singular peak at a delay of $7_{-2}^{+4}$ days and no evidence for a secondary peak. Instead, the singular peak is very broad and high correlation values are found for delays up to $\sim 40$ days.

The overall lags determined by the CCF analysis do not depend significantly on the use of either the purely spectroscopic or the combined spectroscopic and photometric continuum light curve. Both driving light curves recover the same responses of the emission lines with only minor variations of \pm 2 days at maximum. In comparison to the spectroscopic driving light curve, the use of the combined continuum light curve generates more stable CCFs and narrower, more confined CCCDs.

\subsection{Velocity-resolved CCFs of the $H \alpha$ and $H \beta$ line}

In Sect. 3.5 we calculated the time lags of the integrated $\mathrm{H} \alpha$, $\mathrm{H} \beta$, and HeI lines as well as of the central and outer wing regions with respect to the combined V-band continuum at $5180 \AA$ (rest frame). Now, we investigate the profile variations in the $\mathrm{H} \alpha$ and $\mathrm{H} \beta$ lines in more detail by calculating the lags of individual line segments. For the weak HeI $\lambda 5876$ line, the absorption in the central line segment and in the line wings prohibits a clear 

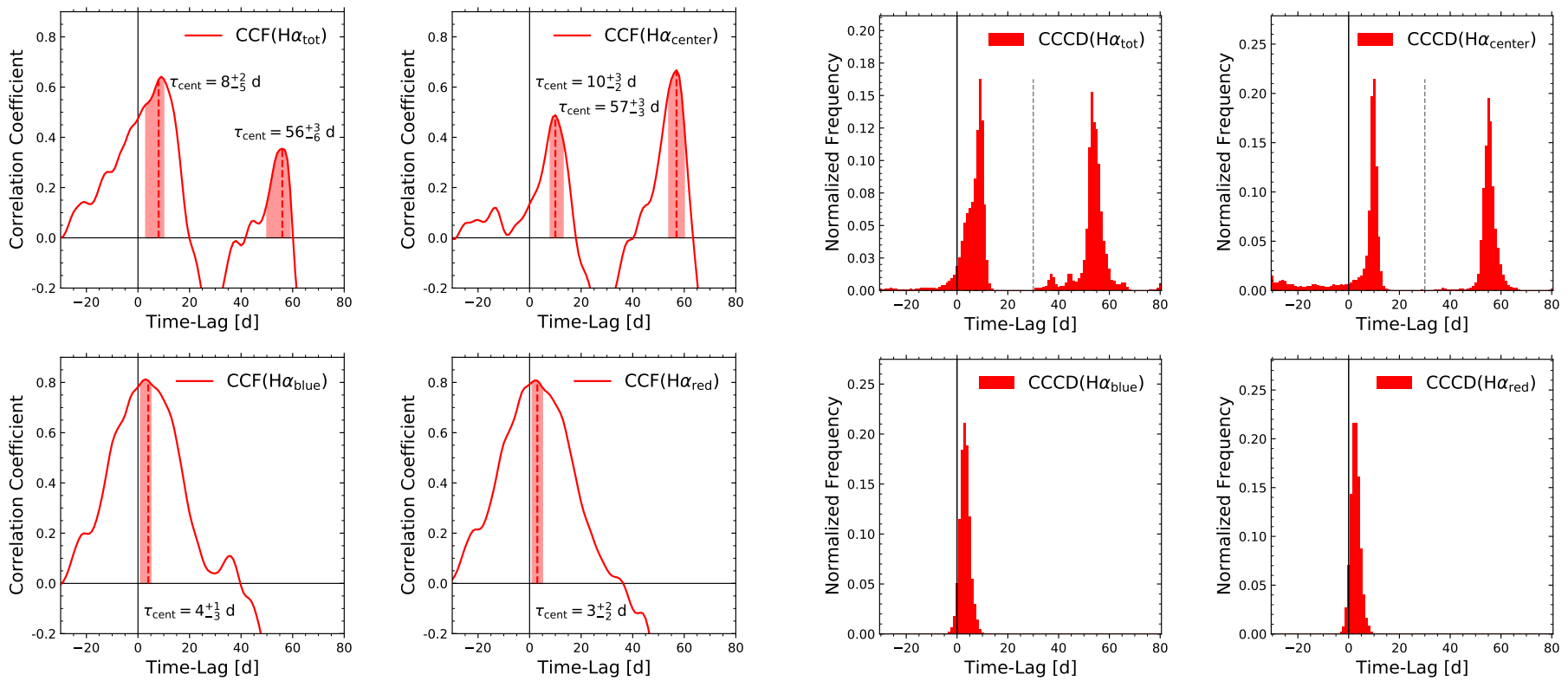

(a)

(b)
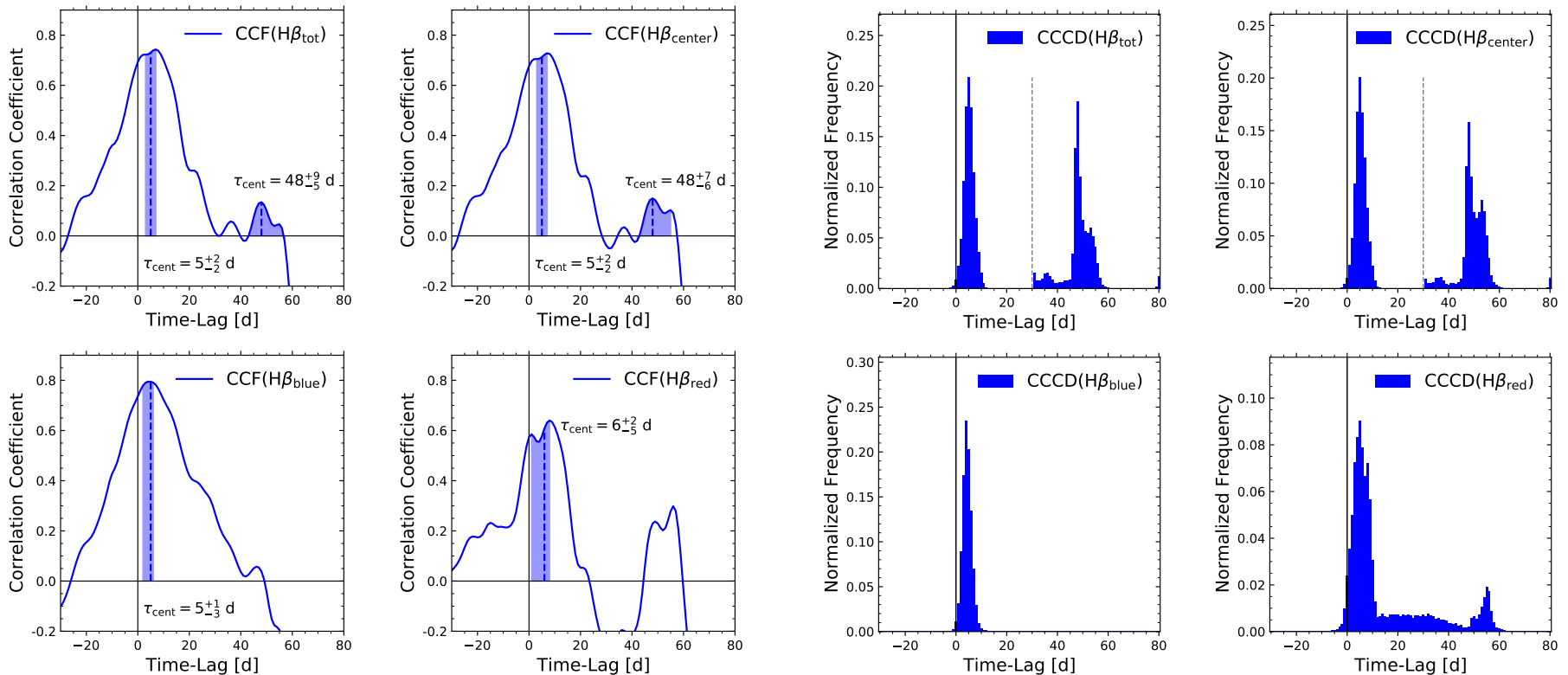

(c)

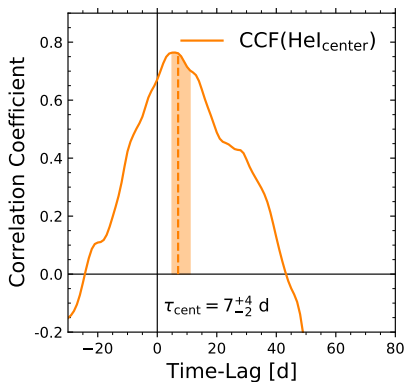

(e)

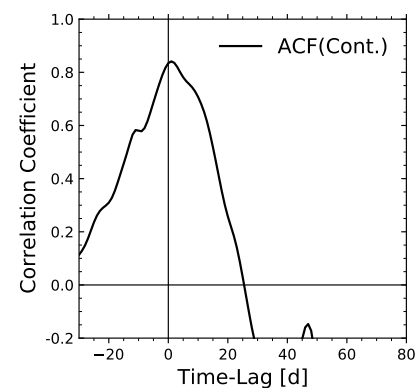

(f)

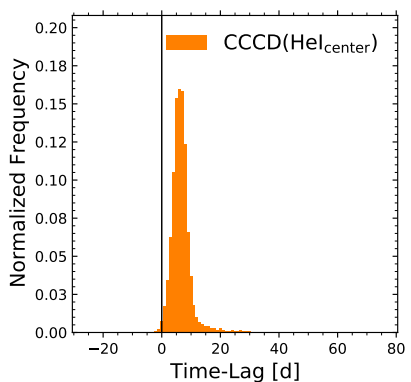

(g) (d)

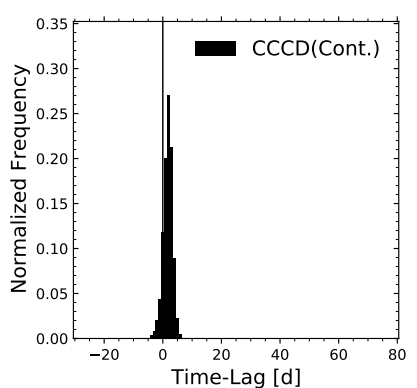

(h)

Fig. 9: Left panel: CCFs of the integrated $\mathrm{H} \alpha(a)$ and $\mathrm{H} \beta(c)$ line, of their segments (center, blue, red), and of the central He $\mathrm{I}$ line $(e)$ with respect to the combined continuum at $5180 \AA$ (rest frame). The time lag, $\tau_{\text {cent }}$, is denoted by a dashed line, with the shaded area corresponding to a $\pm 1 \sigma$ interval. The $\mathrm{CCF}$ of the spectroscopic continuum light curve with respect to the combined (photometric plus spectroscopic) continuum reference light curve is shown in $(f)$. Right panel: CCCDs of the integrated $\mathrm{H} \alpha(b)$ and $\mathrm{H} \beta(d)$ line, of their segments (center, blue, red), and of the central He I line $(g)$ with respect to the combined continuum at $5180 \AA$ (rest frame). The boundary between CCCDs of individual peaks is denoted by a dashed gray line. The CCCD of the spectroscopic continuum light curve with respect to the combined continuum reference light curve is shown in $(h)$. 
Table 7: Cross-correlation lags of the integrated $\mathrm{H} \alpha$ and $\mathrm{H} \beta$ line light curves as well as of their line segments (center, blue, red) with respect to the combined $5180 \AA$ (rest frame) and V-band continuum light curve.

\begin{tabular}{|c|c|c|c|c|c|c|}
\hline \multirow{4}{*}{ Segment } & \multicolumn{6}{|c|}{ Line } \\
\hline & \multicolumn{2}{|c|}{$\mathrm{H} \alpha$} & \multicolumn{2}{|c|}{$\mathrm{H} \beta$} & \multicolumn{2}{|c|}{$\mathrm{He}_{\mathrm{I}}$} \\
\hline & & & & & & \\
\hline & inner & outer & inner & outer & inner & outer \\
\hline total & $8_{-5}^{+2}$ & $56_{-6}^{+3}$ & $5_{-2}^{+2}$ & $48_{-5}^{+9}$ & - & - \\
\hline center & $10_{-2}^{+3}$ & $57_{-3}^{+3}$ & $5_{-2}^{+2}$ & $48_{-6}^{+7}$ & $7_{-2}^{+4}$ & - \\
\hline blue & $4_{-3}^{+1}$ & - & $5_{-3}^{+1}$ & - & - & - \\
\hline red & $3_{-2}^{+2}$ & - & $6_{-5}^{+2}$ & - & - & - \\
\hline
\end{tabular}

determination of a velocity-resolved CCF. The way we proceed has been described before in our studies of line profile variations in Mrk 110 (Kollatschny \& Bischoff 2002; Kollatschny 2003), Mrk 926 (Kollatschny \& Zetzl 2010), 3C120 (Kollatschny et al. 2014) and HE 1136-2304 (Kollatschny et al. 2018).

We sliced the velocity profiles of the continuum-subtracted Balmer lines into velocity segments with a width of $\Delta v=400$ $\mathrm{km} \mathrm{s}^{-1}$. This value of $400 \mathrm{~km} \mathrm{~s}^{-1}$ corresponds to the spectral resolution of our observations. A central line-segment was integrated from $v=-200$ to $+200 \mathrm{~km} \mathrm{~s}^{-1}$. Afterward, we measured the intensities of all subsequent line-of-sight velocity segments from $v=-15800$ to $+15800 \mathrm{~km} \mathrm{~s}^{-1}$ and compiled their light curves. We computed the $\operatorname{CCF}(\tau)$ for each individual linesegment light curve $\left(\Delta v=400 \mathrm{~km} \mathrm{~s}^{-1}\right)$ of $\mathrm{H} \alpha$ and $\mathrm{H} \beta$ with respect to the combined $5180 \AA$ (rest frame) continuum light curve. In this way, we computed velocity-resolved CCFs of $\mathrm{H} \alpha$ and $\mathrm{H} \beta$ as a function of distance to the line center (blue scale). These velocity-resolved CCFs are shown in Figs. 10a and 10b. The green lines in these figures delineate the contour lines of the correlation coefficient at different levels $(0.2,0.4,0.5,0.6$, $0.7,0.75,0.8,0.825)$. The red line shows the rms line profile for comparison.

The $\mathrm{H} \alpha$ line shows two velocity-delay structures in the central segment (within $\pm 5000 \mathrm{~km} \mathrm{~s}^{-1}$ ) at a distance of $57_{-3}^{+3}$ and $10_{-2}^{+3}$ light-days (see Sect. 3.5). Adopting the interpretation of Horne et al. (2021) for the velocity-delay maps of the NGC 5548 STORM campaign, this structure might be interpreted as the upper and lower half of an ellipse in the velocity-delay plane that might be the signature of a line-emitting ring orbiting the black hole $(\mathrm{BH})$ at a radius $R=33.5$ light-days. The stronger far side of the annulus extends to $\tau=(R / c)(1+\sin i) \approx 57$ days, and the weaker near side of the annulus has a delay of $\tau=(R / c)(1-\sin i)$ $\approx 10$ days. This gives $\sin i \sim 0.71$ and thus $i \sim 45^{\circ}$ as the inclination angle of an assumed thin disk.

The $\mathrm{H} \beta$ line also shows two velocity-delay structures in the central segment at $48_{-6}^{+7}$ and $5_{-2}^{+2}$ light-days (see Sect. 3.5). A possible line-emitting ring orbiting the $\mathrm{BH}$ therefore has a radius $R=26.5$ light-days. The weaker far side of the annulus then extends to $\tau=(R / c)(1+\sin i) \approx 48$ days, and the stronger near side of the annulus has a delay at $\tau=(R / c)(1-\sin i) \approx 5$ days. This gives $\sin i \sim 0.81$ and thus $i \sim 54^{\circ}$ of the disk. Therefore, we adopt $i \sim 50^{\circ}$ as the mean disk inclination angle.

\subsection{Central black hole mass}

The masses of the central BHs in AGN can be estimated - based on the assumption that the gas dynamics are dominated by the central massive object - by evaluating

$M=f c \tau \Delta v^{2} G^{-1}$.

The characteristic velocity $\Delta v$ of the emission-line region can be estimated from the FWHM of the rms profiles or from the line dispersions $\sigma_{\text {line }}$. Furthermore, the distance $c \tau$ of the lineemitting regions from the ionizing source can be estimated from the delayed response of the line light curves to the continuum variations. Characteristic distances of the individual lineemitting regions are given by the centroid $\tau_{\text {cent }}$ of the individual CCFs of the emission-line variations relative to the continuum variations (e.g., Koratkar \& Gaskell 1991; Kollatschny \& Dietrich 1997).

The scaling factor $f$ in Eq. 3 is on the order of unity and depends on the kinematics, structure, and orientation of the BLR. This scaling factor differs by $\sim 0.4 \mathrm{dex}$ rms from galaxy to galaxy, for example, depending on whether we see the central accretion disk including the BLR from the edge or face-on. Typically, the scaling factor $f$ is discussed with respect to the line dispersion $\sigma_{\text {line }}(\mathrm{rms})$. Empirically found $f$ values are $f=5.5$ (e.g., Onken et al. 2004), $f=4.31$ (e.g., Grier et al. 2013a), or $f=3.6$ (Graham et al. 2011). The very broad lines in Mrk 926 (see Sect. 3.4) and the high disk inclination angle of $i \sim 50^{\circ}$ (see Sect. 3.6) indicate that Mrk 926's orientation toward us is rather edge-on in comparison to other AGN. Therefore, we adopt an $f$ value of $f=3.6$ in order to calculate the BH mass. We note that the virial factor, $f$, for $\sigma_{\text {line }}$ and FWHM differs since $\mathrm{FWHM} / \sigma_{\text {line }}$ typically takes values of $\sim 2$ (Peterson et al. 2004). We account for that by following the procedure in Kollatschny \& Zetzl (2011, 2013) and, for example, Kollatschny et al. (2018): adopting a correction factor in order to obtain the true rotational velocity, $v$, from the observed FWHM for each line. This reduces the effective $f$ factor of FWHM to $\sim 1.2$. We use FWHM (rms), which generally gives more reliable results than FWHM (mean) due to the fact that the rms profiles only shows the varying fraction of the BLR line-emitting gas. Based on the delays of the central Balmer line regions (see Table 7 and Sect. 3.6) and on the line widths of the rms profiles (FWHM) (see Table 6), we derive a weighted mean $\mathrm{BH}$ mass (see Table 8) of

$M=(1.1 \pm 0.2) \times 10^{8} M_{\odot}$ or $\log \left(M / M_{\odot}\right)=8.04 \pm 0.1$.

The BH mass based on the HeI $\lambda 5876$ line has a larger error due to larger uncertainties in their FWHM. However, it confirms the $\mathrm{BH}$ mass derived from the Balmer lines. The $\mathrm{BH}$ mass estimate based on the $\mathrm{H} \alpha$ line dispersion $\sigma_{\text {line }}$ (mean) is by a factor of $\sim 2$ larger than the BH estimate from the FWHM (rms). Considering the larger uncertainties of $\sigma_{\text {line }}$, it is still in agreement with the $\mathrm{BH}$ mass estimates from FWHM (rms).

\section{Discussion}

\subsection{Optical variability}

\subsubsection{Optical variability amplitudes}

It is known that Mrk926 varied at least since the beginning of the 1990s (Kollatschny et al. 2006). A first variability campaign was carried out during the years 2004-2005 (Kollatschny \& Zetzl 2010). Back then, the optical continuum flux density at $5180 \AA$ varied between 2.4 and $3.6 \times 10^{-15} \mathrm{erg} \mathrm{cm}^{-2} \mathrm{~s}^{-1} \AA^{-1}$. In July 


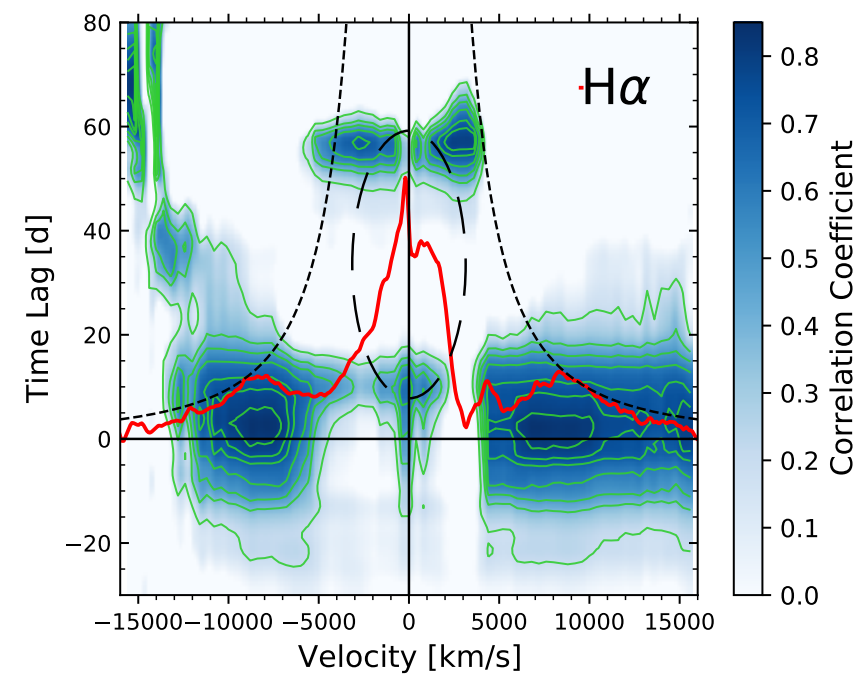

(a)

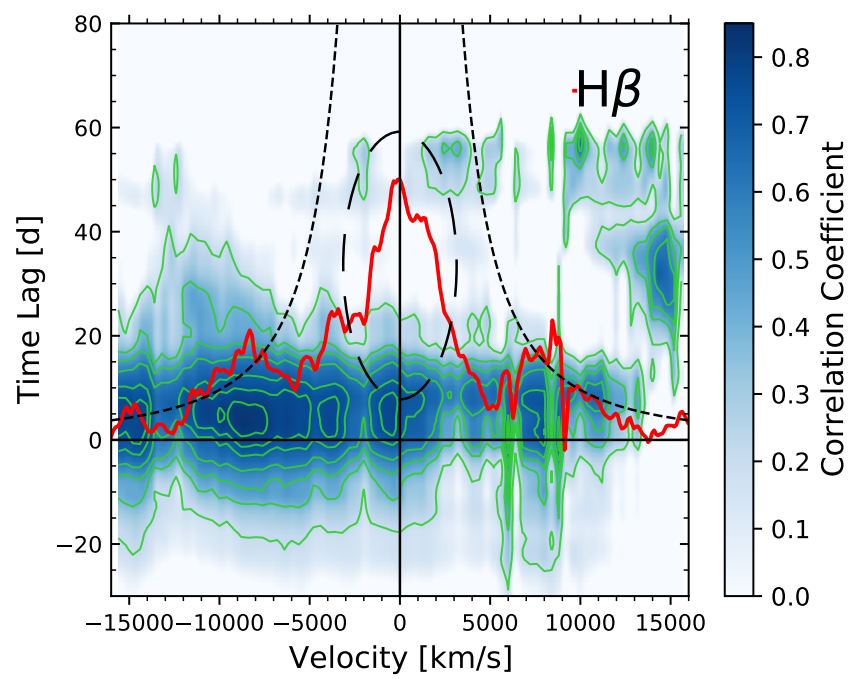

(b)

Fig. 10: Velocity-resolved $\operatorname{CCFs}(\tau, v)$ showing the correlation coefficient of the $\mathrm{H} \alpha(a)$ and $\mathrm{H} \beta(b)$ line segment light curves $(\Delta v \sim$ $400 \mathrm{~km} \mathrm{~s}^{-1}$ ) with respect to the combined continuum light curve as a function of velocity and time delay (blue scale). Contours of the correlation coefficients are plotted at levels $0.2,0.4,0.5,0.6,0.7,0.75,0.8$, and 0.825 (green lines). In each plot, the rms profile of the emission line is shown in red. The dashed curve shows the escape velocities for a Keplerian disk inclined by $\sim 50^{\circ}$ with a central mass of $1.1 \times 10^{8} M_{\odot}$. The dashed ellipse corresponds to a circular Keplerian orbit at $R / c=33.5 \mathrm{~d}$ inclined by $\sim 50^{\circ}$ to the line of sight mapped onto the velocity-delay plane. The line center $\left(v=0 \mathrm{~km} \mathrm{~s}^{-1}\right)$ and the time delay of $\tau=0$ days are indicated by a vertical and a horizontal black line, respectively. The delays computed for the red wing of $\mathrm{H} \beta$ are disturbed by the [O III] $\lambda$ 4959, 5007 lines, which results in higher-delay residuals not confined within the viral envelope.

Table 8: Black hole masses based on the FWHM of the line profiles in the rms spectra and the line dispersion $\sigma_{\text {line }}$ of the mean $\mathrm{H} \alpha$ profile. The masses were calculated assuming an $f$ value of 3.6.

\begin{tabular}{lcc}
\hline \hline Line & $\begin{array}{c}M_{\mathrm{BH}, \mathrm{FWHM}} \\
{\left[10^{8} M_{\odot}\right]}\end{array}$ & $\begin{array}{c}M_{\mathrm{BH}, \sigma_{\text {line }}} \\
{\left[10^{8} M_{\odot}\right]}\end{array}$ \\
\hline $\mathrm{H} \alpha$ & $1.2 \pm 0.4$ & $2.8 \pm 1.7$ \\
$\mathrm{H} \beta$ & $1.1 \pm 0.3$ & - \\
\hline weigh. mean & $1.1 \pm 0.2$ & - \\
\hline \hline HeI $\lambda 5876$ & $1.0 \pm 0.6$ & - \\
\hline
\end{tabular}

2010, the continuum flux density was significantly higher and amounted to a value of $6 \times 10^{-15} \mathrm{erg} \mathrm{cm}^{-2} \mathrm{~s}^{-1} \AA^{-1}$ at $5180 \AA$ (rest frame) at the beginning of the variability campaign presented here. Immediately afterward, the optical continuum started to drop, finally reaching less than $50 \%$ of its original intensity (see Fig. 5 and Table B.1) within only 2.5 months. Even more remarkably, the true non-stellar continuum in Mrk 926 declines to only $30-40 \%$ continuum intensity between the beginning and end of the variability campaign taking into account the constant host galaxy contribution to the flux as determined by means of the FVG method (see Table 4).

The variability amplitude in the blue spectral range is higher than in the red (see Table 5 and the rms spectrum in Fig. 3). Interestingly, the continuum variation amplitudes in Mrk 926 are much higher than those observed in other highly variable and even changing-look AGN. For example, during a post-outburst campaign in 2014-2015, the changing-look AGN HE 1136-2304 exhibited a fractional variation of $F_{\mathrm{V}, \mathrm{var}}=0.11$ and $F_{\mathrm{V} \text {,var }}=0.25$ with and without host, respectively (Zetzl et al. 2018). Mrk 926 exhibits fractional variation of $F_{\mathrm{V} \text {,var }}=0.22$ and $F_{\mathrm{V} \text {,var }}=0.31$ with and without host, respectively. The fractional variation depends on the duration of the monitoring campaign, on the examined wavelength, and on the (accurate) subtraction of the host galaxy contribution. A typical value for the fractional variation $F_{\mathrm{V} \text {,var }}$ of the continuum at roughly $5100 \AA$ is 0.05 to 0.15 for variability periods of 6 to 12 months. For longer campaigns, typical $F_{\mathrm{V} \text {,var }}$ values are higher and range from 0.1 to 0.25 (Zetzl et al. 2018, and references therein). On timescales of years, a decline of the continuum to only $20 \%$ of its original value has been observed for some sources (e.g., Fairall 9; Kollatschny \& Fricke 1985). However, Mrk 926 was observed for a much shorter period and showed a similarly drastic decline. To summarize, it can be stated that the optical continuum variations in Mrk 926 are very strong.

\subsubsection{Signature of an accretion disk in Mrk 926}

We now intend to test whether the observed continuum spectral index in the optical is consistent with theoretical predictions from the Shakura \& Sunyaev accretion disk model (SS73; Shakura \& Sunyaev 1973). Specifically, the Shakura \& Sunyaev model predicts $f_{v} \propto v^{1 / 3}$ over the self-similar part of the spectrum, that is, far away from the spectral bands affected by the inner (UV to extreme UV) and outer (far-infrared) disk radii. This holds for Mrk 926 over the optical range. The spectral in$\operatorname{dex}$ of $\alpha=1 / 3$ for $f_{v}$ then translates to $\beta=-7 / 3$ for $f_{\lambda}$ as $f_{\lambda}=f_{v} d v / d \lambda \sim v^{1 / 3} v^{2} \sim \lambda^{-7 / 3}$. 
Figure 11 shows the observed rms spectrum corrected for Galactic foreground extinction. A power-law model $F_{\lambda} \propto \lambda^{-\beta}$ was fitted to the continuum (blue shaded areas), giving a spectral index $\beta=2.33 \pm 0.02$. The rms spectrum of the continuum is thus consistent with a power- law with a spectral index of $\beta=7 / 3$ as predicted by the Shakura \& Sunyaev model.

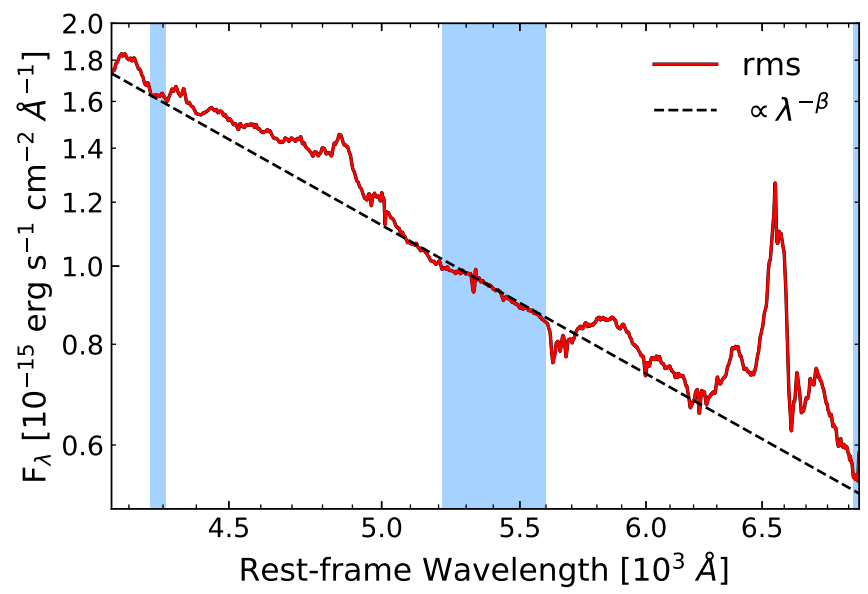

Fig. 11: RMS spectrum of the HET campaign corrected for Galactic foreground extinction. A power-law model was fitted to the continuum, giving a spectral index $\beta=2.33 \pm 0.02$.

\subsubsection{Balmer decrement variability}

The Balmer decrement $F\left(\mathrm{H} \alpha_{\text {narrow }}\right) / F\left(\mathrm{H} \beta_{\text {narrow }}\right)$ of the narrowline components in Mrk 926 amounts to 2.70 (see Table 3). This corresponds to the expected theoretical line ratio (Case B) without any reddening. The lack of reddening toward the narrowline region (NLR) is consistent with the insignificant reddening implied by the RMS fit to a Shakura \& Sunyaev model (see Sect. 4.1.2). In contrast to the constant Balmer decrement of the narrow lines, the Balmer decrement of the broad-line components takes values of 3.4 to 4.2. Figure 12 shows the Balmer decrement $F\left(\mathrm{H} \alpha_{\text {broad }}\right) / F\left(\mathrm{H} \beta_{\text {broad }}\right)$ versus broad-line $\mathrm{H} \beta$ flux.

The Balmer decrement varies as a function of the broad $\mathrm{H} \beta$ flux. More precisely, the Balmer decrement shows a roughly linear dependence on the $\mathrm{H} \beta$ flux, and increases with decreasing $\mathrm{H} \beta$ broad-line intensity. Similar, although even stronger variations in the Balmer decrement have been found before in highly variable Seyfert galaxies as, for example, NGC 7603 (Kollatschny et al. 2000) or HE 1136-2304 (Kollatschny et al. 2018). The broad-line Balmer decrement in these galaxies increased up to values of 7.3 in combination with decreasing $\mathrm{H} \beta$ line intensities. These observations might be explained by optical depth effects in the BLR. This is in accordance with the finding that $\mathrm{H} \alpha$ often originates at larger distances than $\mathrm{H} \beta$. It has been discussed by Korista \& Goad (2004) that the radial stratification in the BLR is a result of optical-depth effects of the Balmer lines: the broad-line Balmer decrement decreases in high continuum states and increases in low states.

\section{2. $H \beta$ lag versus optical continuum luminosity}

Now, we test whether Mrk 926 follows the general trend of the $\mathrm{H} \beta$-lag and optical continuum luminosity relationship $\left(\mathrm{R}_{\mathrm{BLR}}-\right.$ $\mathrm{L}_{\mathrm{AGN}}$ ) for AGN (e.g., Kaspi et al. 2000; Bentz et al. 2013;

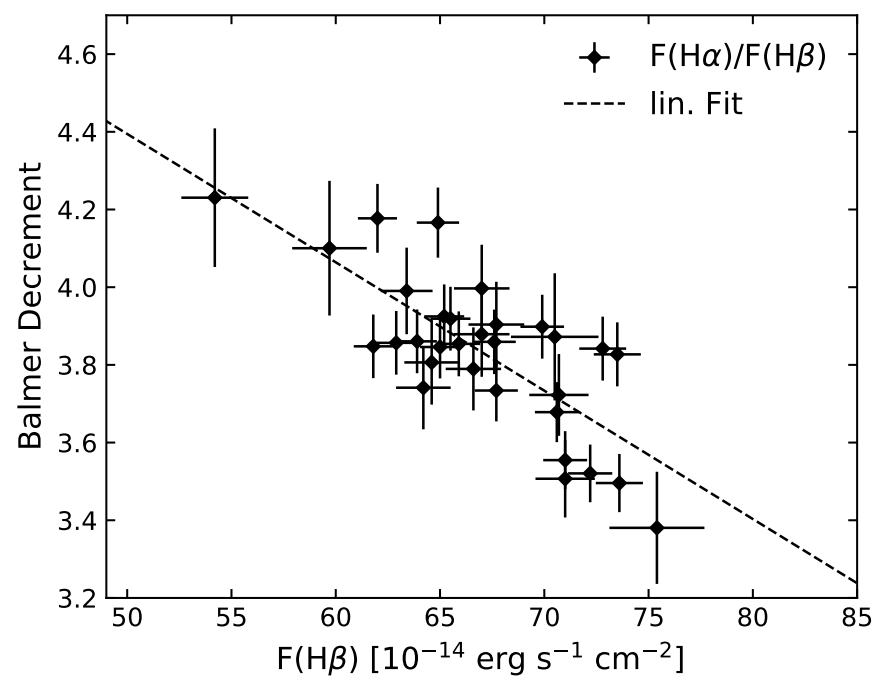

Fig. 12: Balmer decrement, $F(\mathrm{H} \alpha) / F(\mathrm{H} \beta)$, of the broad-line components versus broad-line $\mathrm{H} \beta$ intensity. The dashed line on the graph represents the linear regression.

Kilerci Eser et al. 2015; Grier et al. 2017; Kollatschny et al. 2018, and references therein). We determined continuum luminosities $\log \left(\lambda L_{\lambda} / \mathrm{erg} \mathrm{s}^{-1}\right)$ of 43.68 to 44.13 (1.87 to 5.30 $\times 10^{-15} \mathrm{erg} \mathrm{s}^{-1} \mathrm{~cm}^{-2} \AA^{-1}$ ) in the optical at $5180 \AA$ (rest frame) after correction for the contribution of the host galaxy. Furthermore, we derived a mean distance of 26.5 light-days for the $\mathrm{H} \beta$ line-emitting region based on the delay of the $\mathrm{H} \beta$ line variability with respect to the optical continuum light curve. This derived distance of 26.5 light-days for the $\mathrm{H} \beta$ line-emitting region and the observed continuum luminosities closely follows the general $R_{\mathrm{BLR}}-L_{\mathrm{AGN}}$ relation with a slope of $\alpha \sim 0.53$ (Bentz et al. 2013).

\subsection{Line profiles}

\subsubsection{Mean and rms line profiles of the 2010 campaign}

The mean and rms profiles of the Balmer and He I $\lambda 5876$ lines in velocity space are presented in Fig. 8 . The mean and rms profiles of $\mathrm{H} \alpha$ and $\mathrm{H} \beta$ are very similar. They exhibit line widths (FWHM) of $\sim 5250 \mathrm{~km} \mathrm{~s}^{-1}$ (mean profiles) and line widths of $\sim 4000 \mathrm{~km} \mathrm{~s}^{-1}$ (rms profiles) (see Table 6). These values are larger than the average. The H $\alpha$ FWHM distribution for broadline quasars - based on many thousand emission-line galaxies from the Sloan Digital Sky Survey - shows a clear maximum at about $2000 \mathrm{~km} \mathrm{~s}^{-1}$ and falls steadily to FWHM values of about $9000 \mathrm{~km} \mathrm{~s}^{-1}$ (Hao et al. 2005). There is an additional inner red emission component to be seen at $\sim+1500 \mathrm{~km} \mathrm{~s}^{-1}$. Such inner red components have been seen before in other variable AGN, for example Ark 120 (Kollatschny et al. 1981), NGC4593 (Kollatschny \& Dietrich 1997), NGC7603 (Kollatschny et al. 2000), or HE 1136-2304 (Kollatschny et al. 2018). Possible explanations for these red components might be disk components, additional radial motions, or opacity components. More comments regarding inner red line components can be found, for example, in Gaskell \& Harrington (2018) or Du et al. (2018).

The Balmer lines show symmetric additional outer-line components between \pm 5000 and $\pm 13000 \mathrm{~km} \mathrm{~s}^{-1}$, which we term Balmer satellites. We note that the red wing of He I $\lambda 5876$ might 
also indicate the presence of such a line satellite; however, due to the interference by absorption, a clear detection cannot be claimed. In particular, the Balmer satellites components show up in the rms profiles and their relative variability amplitudes are stronger than that of the central components between -5000 and $+5000 \mathrm{~km} \mathrm{~s}^{-1}$. The fractional variations in the individual line components are given in Table 5. Five years before the variability campaign presented here, when Mrk 926 was by 50\% fainter in the continuum compared to the beginning of our campaign, these outer components in the rms profiles were also present, but are now slightly more pronounced (Kollatschny \& Zetzl 2010). Back in 2005 , the central line components were noticeably broader when the source was in a lower state. The central Balmer line components exhibited FWHM rms of roughly $8500 \mathrm{~km} \mathrm{~s}^{-1}$ in 2005 in comparison to $4000 \mathrm{~km} \mathrm{~s}^{-1}$ in 2010 . The uncertainties in the line dispersion $\sigma_{\text {line }}$ of the broad emission lines determined in Sect. 3.4 are relatively high. Generally, the line dispersion $\sigma_{\text {line }}$ is very sensitive to the line widths used for integration. However, in Mrk 926, the uncertainties in the line widths - full width at zero intensity (FWZI) - are high because of the large line widths and the additional outer line-components. Therefore, we did not derive a FWHM $/ \sigma$ to determine the height/radius ratio of the BLR as presented in other galaxies (e.g., Kollatschny et al. 2018). Typical values for FWHM/ $\sigma$ in other galaxies are on the order of two or more. Only in very rare cases the $\sigma_{\text {line }}$ values are equal or larger than the FWHM values (Peterson et al. 2004; Kollatschny \& Zetzl 2011, 2013).

\subsubsection{Comparison of the 2010 and $2004-2005$ campaigns}

We now compare the Balmer line profiles observed in 2010 with the Balmer line profiles observed during the campaign in 20042005. First, the continuum as well as broad-line flux in 2005 was by a factor of about two smaller than in 2010 (Kollatschny \& Zetzl 2010). Second, the comparison of the Balmer line profiles also reveals differences in the mean and rms profiles. A strong blue component was present in the mean profiles in 2005 , but nearly disappeared in 2010. A small residual of this blue component appears to be left in the rms profiles at around -1500 $\mathrm{km} \mathrm{s}^{-1}$. The narrow-line subtraction for the campaign of 2010 revealed a red component in the mean profile of $\mathrm{H} \alpha$ at around $\pm 1200 \mathrm{~km} \mathrm{~s}^{-1}$ (see Sect. 3.1) that is also discernible in the $\mathrm{H} \beta$ profile (see above). Moreover, the rms profiles show a variable red component with the same velocity. This inner red component is in agreement with a red and slightly stronger component already present in the mean and rms profiles of the campaign in 2004/05. In summary, both the mean and rms profiles of the campaigns in 2004/05 and 2010 show blue and red components at mirrored velocities. These components were stronger in 2004/05. Presumably, these symmetric (with respect to lineof-sight velocity) components are the signature of an accretion disk as accretion disks are expected to result in double-peaked profiles (e.g., Horne \& Marsh 1986; Eracleous \& Halpern 2003; Gezari et al. 2007; Shapovalova et al. 2013; Storchi-Bergmann et al. 2017, and references therein).

The highly variable outer rms Balmer satellite components were also present in 2005, but are more pronounced in 2010. Such variable outer line-components are very rare and have been seen only in the rms profiles of a few broad-line radio galaxies, for example, 3C390.3, Arp102B, 3C382, or 3C332 (Gezari et al. 2007). Mrk 926 shows a radio flux of $18 \pm 5 \mathrm{mJy}$ at $5 \mathrm{Ghz}$ (Bicay et al. 1995). The optical B-band flux is $6.6 \mathrm{mJy}$ (McAlary et al. 1983). Therefore, the radio-to-optical luminosity $\mathrm{R}(\mathrm{F}(5$ $\mathrm{Ghz}) / \mathrm{F}(\mathrm{B}-\mathrm{band})$ ) has a value of 3 . This is above the definition for a radio-quiet AGN (R: $0.1-1)$ and below the value for a radio-loud AGN (R: 10 - 1000) (Kellermann et al. 1989). However, the highly variable outer components in the rms profiles shown by Gezari et al. (2007) are almost exclusively connected to the flanks of the double-peaked mean profiles, which are assumed to originate from an accretion disk. This is not the case for Mrk 926. Instead, the rms components are further out and their position overlaps with the outer line-wings, but not the lineflanks (see Fig. 8).

\subsection{Central black hole mass and Eddington ratio, $L / L_{e d d}$}

In Sect. 3.7, we derived a central BH mass of $1.1 \times 10^{8} M_{\odot}$. This is a normal $\mathrm{BH}$ mass in the typical range of $10^{6}-10^{9} M_{\odot}$ (e.g., Woo \& Urry 2002; Peterson et al. 2004). In the following, we derive the Eddington ratios, $L / L_{\text {edd }}$, for the low and high intensity state of Mrk 926 during our variability campaign. The optical luminosity of Mrk 926 during the low state in November 2010 was by a factor of 2.8 lower with respect to the high state in August 2010. We derive bolometric luminosities of $L_{\text {bol }}(\max )=$ $1.2 \times 10^{45} \mathrm{erg} \mathrm{s}^{-1}$ and $L_{\mathrm{bol}}(\mathrm{min})=4.3 \times 10^{44} \mathrm{erg} \mathrm{s}^{-1}$ for the high and low state, respectively, based on the monochromatic luminosity $\mathrm{L}_{5100}$ and on an average bolometric correction factor of $f_{\text {bol }}=L_{\text {bol }} / L_{5100}=10$ (Kaspi et al. 2000; Castelló-Mor et al. 2016). Although the boundary between Seyfert galaxies and the higher luminosity quasars is not well defined, a bolometric luminosity of $L_{\text {bol }}=10^{45} \mathrm{erg} \mathrm{s}^{-1}$ of the central source is often used as a dividing line to distinguish between these two types (Netzer 2013). This places Mrk 926 directly at the dividing line, hence being, depending on the time of observation, either a very strong Seyfert 1 galaxy or a quasar.

The $\mathrm{BH}$ mass of $1.1 \times 10^{8} M_{\odot}$ corresponds to an Eddington luminosity of $L_{\text {edd }}=1.4 \times 10^{46} \mathrm{erg} \mathrm{s}^{-1}$. Therefore, the Eddington ratios, $L / L_{\text {edd }}$, for the high and low state have comparatively low values, 8 and 3 percent only. Such low Eddington ratios of a few percent are typical for highly variable objects. For example, the changing-look AGN IRAS 23226-3843 showed a low Eddington ratio of only one percent (Kollatschny et al. 2020). This low Eddington ratio is consistent with investigations of Noda \& Done (2018) and MacLeod et al. (2019). In particular, Noda \& Done (2018) suggested that all changing-look AGN are associated with state transitions at Eddington ratios of a few percent.

\subsection{Structure and kinematics of the BLR}

\subsubsection{CCFs of the central, blue, and red line segments}

The CCFs of the integrated Balmer line light curves and of the central Balmer line component light curves (within \pm 5000 $\mathrm{km} \mathrm{s}^{-1}$ ) exhibit two discrete peaks, one at a delay of $\sim 10$ days $(\mathrm{H} \alpha)$ and $\sim 5$ days $(\mathrm{H} \beta)$, and the outer at a delay of $\sim 56$ days $(\mathrm{H} \alpha)$ and $\sim 48$ days $(\mathrm{H} \beta)$. The long-delay peaks of $\mathrm{H} \alpha$ and $\mathrm{H} \beta$ indicate a stratification of the BLR as the CCFs peak at $57_{-3}^{+3}$ and $48_{-6}^{+7}$ days, respectively. The blue and red Balmer segment light curves (i.e., the light curves of the Balmer satellite components) are single-peaked, with delays of $\sim 4$ days for $\mathrm{H} \alpha$ and $\sim 5$ days for $\mathrm{H} \beta$. This means that the outer wings respond promptly to the variations in the continuum.

The HeI $\lambda 5876$ line shows no double-peaked CCF. Instead, the CCF of the central line profile light curve shows a very broad peak with high correlations up to $\sim 40$ days. This broad peak might be caused by two merging peaks (i.e., by two independently responding components exactly as observed for $\mathrm{H} \alpha$ and 
$\mathrm{H} \beta$ ) but with relatively broad transfer functions that our campaign was not able to resolve.

\subsubsection{Velocity-resolved CCFs of the Balmer lines}

The velocity-resolved CCFs of $\mathrm{H} \alpha$ and $\mathrm{H} \beta$ (Figs. 10a and 10b) are, in essence, similar to each other. The delays of the central profiles and of the outer wings are all confined within the virial envelope for a Keplerian disk inclined by $\sim 50^{\circ}$ with a central mass of $1.1 \times 10^{8} M_{\odot}$. Signatures outside of the virial envelopes can be attributed to relatively strong narrow-line residuals (e.g., in the red wing of $\mathrm{H} \beta$ ), to overlapping velocity-resolved line signatures (e.g., of $\mathrm{He}$ II $\lambda 4686$ and $\mathrm{H} \beta$ ), or to strong absorption disturbing the segment light curves (e.g., in the outermost blue wing of $\mathrm{H} \alpha$ ). Both velocity-resolved CCFs show a broad inner delay region with small time lags of $\sim 3-10$ days. The correlation is strongest in the line wings beyond $\pm 5000 \mathrm{~km} \mathrm{~s}^{-1}$, exactly where we observed the maximum of the additional, varying Balmer satellites in the rms profiles. These Balmer satellites respond promptly to the continuum variations with delays of only $\sim 3-5$ days.

The $\mathrm{H} \alpha$ line shows two velocity-delay structures in the central segment (within $\pm 5000 \mathrm{~km} \mathrm{~s}^{-1}$ ) at $10_{-2}^{+3}$ and $57_{-3}^{+3}$ light-days. This might be interpreted as the upper and lower half of an ellipse in the velocity-delay plane, which might be the signature of a line-emitting ring orbiting the $\mathrm{BH}$ at a radius $R=33.5$ light-days. This interpretation of the two velocity-delay structures being the signature of a line-emitting ring is analogous to that of the velocity-delay maps of NGC 5548 based on the STORM campaign (Horne et al. 2021), and is in turn supported by our finding that the line profiles in Mrk 926 show the signature of an accretion disk (see Sect. 4.3). Likewise, the $\mathrm{H} \beta$ line shows two velocity-delay structures in the central region at $5_{-2}^{+2}$ and $48_{-6}^{+7}$ light-days, although less pronounced. The possible line-emitting ring orbiting the $\mathrm{BH}$ has a radius of $R=26.5$ lightdays. However, the relative strength of the responses of the near and far side of the ring is different in $\mathrm{H} \alpha$ and $\mathrm{H} \beta$. This might be caused by optical depths effects. Moreover, $\mathrm{H} \alpha$ shows a strong discontinuity between the signature of the central line profile and the Balmer satellites, which is less pronounced in the velocityresolved CCF of $\mathrm{H} \beta$. This might be due to interference of $\mathrm{H} \beta$ with the He II $\lambda 4686$ line.

Assuming the two Balmer velocity-delay structures to be the signature of an inclined accretion disk, we determined a mean inclination angle of $i \sim 50^{\circ}$. This rather high inclination angle is supported by the remarkably broad emission lines $\left(\sim 30000 \mathrm{~km} \mathrm{~s}^{-1}\right.$ FWZI) in Mrk 926.

\subsubsection{Origin of the Balmer satellites}

The existence of the additional, fast-response Balmer satellite components in the rms line profiles and in the velocity-resolved CCFs is an indication for an additional line-emitting component in the nuclear region that might not be directly connected to the accretion disk. Because of the clear separation between the central and outer components, their differing variability behavior, the clearly distinct time lags, as well as the outer rms component not being connected to the line-flanks, we propose that the Balmer satellite components may originate in a region that is spatially distinct from the rest of the BLR. For example, the outer wing components might be connected with a different spatial region like a hollow outflow cone with a high tilt angle or small-scale central radio jets. Notably, Ulvestad \& Wilson
(1984) found a slightly resolved radio source for Mrk 926 with the VLA at $6 \mathrm{~cm}$. Mundell et al. (2000) suggested additional extended radio emission on scales between about 2 and 260 mas based on observations with the VLBA and VLA and the comparison of their radio beams.

\section{Summary}

We present results of a spectroscopic and photometric monitoring campaign of the very broad-line AGN Mrk 926, which was carried out with the 10m HET telescope and the Wise Observatory in 2010. Our findings can be summarized as follows:

(1) Mrk 926 is a highly variable AGN. The continuum luminosity showed a drastic decrease during our campaign. It dropped to less than $50 \%$ of its original luminosity within only 2.5 months.

(2) Mrk 926 shows very broad $\mathrm{H} \alpha$ and $\mathrm{H} \beta$ line profiles, with additional outer Balmer satellite components ranging from \pm 5000 to $\pm 13000 \mathrm{~km} \mathrm{~s}^{-1}$ that are clearly discernible in the rms spectra.

(3) The Balmer lines show two velocity-delay structures in their central line component (within $\pm 5000 \mathrm{~km} \mathrm{~s}^{-1}$ ), at $\sim 10$ and $\sim 57$ light-days $(\mathrm{H} \alpha)$ and at $\sim 5$ and $\sim 48$ light-days $(\mathrm{H} \beta)$. These structures might be interpreted as the upper and lower halves of an ellipse in the velocity-delay plane, which might be the signature of a line-emitting ring orbiting the $\mathrm{BH}$ at radii, $R$, of 33.5 and 26.5 light-days.

(4) The continuum luminosities $\log \left(\lambda L_{\lambda} / \mathrm{erg} \mathrm{s}^{-1}\right)$ at $5180 \AA$ (rest frame) of 43.68 to 44.13 are in good agreement with the established $R_{\mathrm{BLR}}-L_{\mathrm{AGN}}$ relation.

(5) The derived $\mathrm{BH}$ mass of $1.1 \times 10^{8} M_{\odot}$ indicates a low Eddington ratio, which decreased from 8 to 3 percent within only 2.5 months.

(6) Based on the comparison of the variability behavior of the central line component and the outer line wings, we speculate that the outer emission components (the Balmer satellites) originate in a different, spatially distinct region.

Further densely sampled spectroscopic and photometric studies of this highly variable AGN are desirable.

Acknowledgements. This work has been supported by the DFG grants KO857/35-1 and CH71/34-3. KH acknowledges support from STFC grant ST/M001296/1. DC acknowledges support from ISF grant 2398/19. This paper is based on observations obtained with the Hobby-Eberly Telescope, which is a joint project of the University of Texas at Austin, the Pennsylvania State University, Ludwig-Maximilians-Universität München, and Georg-August-Universität Göttingen.

\section{References}

Ahumada, R., Prieto, C. A., Almeida, A., et al. 2020, ApJS, 249, 3 Barth, A. J., Pancoast, A., Thorman, S. J., et al. 2011, ApJ, 743, L4 Bentz, M. C., Denney, K. D., Grier, C. J., et al. 2013, ApJ, 767, 149 Bentz, M. C., Walsh, J. L., Barth, A. J., et al. 2008, ApJ, 689, L21 Bessell, M. S. 1990, PASP, 102, 1181

Bicay, M. D., Kojoian, G., Seal, J., Dickinson, D. F., \& Malkan, M. A. 1995, ApJS, 98, 369

Brosch, N., Polishook, D., Shporer, A., et al. 2008, Ap\&SS, 314, 163 Castelló-Mor, N., Netzer, H., \& Kaspi, S. 2016, MNRAS, 458, 1839 Choloniewski, J. 1981, Acta Astron., 31, 293

Denney, K. D., Peterson, B. M., Pogge, R. W., et al. 2009, ApJ, 704, L80

Du, P., Brotherton, M. S., Wang, K., et al. 2018, ApJ, 869, 142

Durret, F. \& Bergeron, J. 1988, A\&AS, 75, 273

Edelson, R., Turner, T. J., Pounds, K., et al. 2002, ApJ, 568, 610

Edelson, R. A. \& Krolik, J. H. 1988, ApJ, 333, 646

Eracleous, M. \& Halpern, J. P. 2003, ApJ, 599, 886 
Fromerth, M. J. \& Melia, F. 2000, ApJ, 533, 172

Garnier, R., Paturel, G., Petit, C., Marthinet, M. C., \& Rousseau, J. 1996, A\&AS, 117,467

Gaskell, C. M. \& Harrington, P. Z. 2018, MNRAS, 478, 1660

Gezari, S., Halpern, J. P., \& Eracleous, M. 2007, ApJS, 169, 167

Graham, A. W., Onken, C. A., Athanassoula, E., \& Combes, F. 2011, MNRAS, 412, 2211

Grier, C. J., Martini, P., Watson, L. C., et al. 2013a, ApJ, 773, 90

Grier, C. J., Peterson, B. M., Horne, K., et al. 2013b, ApJ, 764, 47

Grier, C. J., Peterson, B. M., Pogge, R. W., et al. 2012, ApJ, 755, 60

Grier, C. J., Trump, J. R., Shen, Y., et al. 2017, ApJ, 851, 21

Haas, M., Chini, R., Ramolla, M., et al. 2011, A\&A, 535, A73

Hao, L., Strauss, M. A., Tremonti, C. A., et al. 2005, AJ, 129, 1783

Horne, K., De Rosa, G., Peterson, B. M., et al. 2021, ApJ, 907, 76

Horne, K. \& Marsh, T. R. 1986, MNRAS, 218, 761

Kaspi, S., Maoz, D., Netzer, H., et al. 2005, ApJ, 629, 61

Kaspi, S., Smith, P. S., Netzer, H., et al. 2000, ApJ, 533, 631

Kellermann, K. I., Sramek, R., Schmidt, M., Shaffer, D. B., \& Green, R. 1989, AJ, 98, 1195

Kilerci Eser, E., Vestergaard, M., Peterson, B. M., Denney, K. D., \& Bentz, M. C. 2015, ApJ, 801, 8

Kollatschny, W. 2003, A\&A, 407, 461

Kollatschny, W. \& Bischoff, K. 2002, A\&A, 386, L19

Kollatschny, W., Bischoff, K., \& Dietrich, M. 2000, A\&A, 361, 901

Kollatschny, W., Bischoff, K., Robinson, E. L., Welsh, W. F., \& Hill, G. J. 2001, A\&A, 379, 125

Kollatschny, W. \& Dietrich, M. 1997, A\&A, 323, 5

Kollatschny, W. \& Fricke, K. J. 1985, A\&A, 146, L11

Kollatschny, W., Fricke, K. J., Schleicher, H., \& Yorke, H. W. 1981, A\&A, 102, L23

Kollatschny, W., Grupe, D., Parker, M. L., et al. 2020, A\&A, 638, A91

Kollatschny, W., Ochmann, M. W., Zetzl, M., et al. 2018, A\&A, 619, A168

Kollatschny, W., Ulbrich, K., Zetzl, M., Kaspi, S., \& Haas, M. 2014, A\&A, 566, A106

Kollatschny, W. \& Zetzl, M. 2010, A\&A, 522, A36

Kollatschny, W. \& Zetzl, M. 2011, Nature, 470, 366

Kollatschny, W. \& Zetzl, M. 2013, A\&A, 549, A100

Kollatschny, W., Zetzl, M., \& Dietrich, M. 2006, A\&A, 454, 459

Koratkar, A. P. \& Gaskell, C. M. 1991, ApJ, 370, L61

Korista, K. T. \& Goad, M. R. 2004, ApJ, 606, 749

MacLeod, C. L., Green, P. J., Anderson, S. F., et al. 2019, ApJ, 874, 8

McAlary, C. W., McLaren, R. A., McGonegal, R. J., \& Maza, J. 1983, ApJS, 52, 341

Morris, S. L. \& Ward, M. J. 1988, MNRAS, 230, 639

Mundell, C. G., Wilson, A. S., Ulvestad, J. S., \& Roy, A. L. 2000, ApJ, 529, 816

Netzer, H. 2013, The Physics and Evolution of Active Galactic Nuclei

Netzer, H., Heller, A., Loinger, F., et al. 1996, MNRAS, 279, 429

Noda, H. \& Done, C. 2018, MNRAS, 480, 3898

Onken, C. A., Ferrarese, L., Merritt, D., et al. 2004, ApJ, 615, 645

Osterbrock, D. E. \& Shuder, J. M. 1982, ApJS, 49, 149

Peterson, B. M., Berlind, P., Bertram, R., et al. 2002, ApJ, 581, 197

Peterson, B. M., Ferrarese, L., Gilbert, K. M., et al. 2004, ApJ, 613, 682

Peterson, B. M., Wanders, I., Bertram, R., et al. 1998, ApJ, 501, 82

Rehfeld, K., Marwan, N., Heitzig, J., \& Kurths, J. 2011, Nonlinear Processes in Geophysics, 18, 389

Rodríguez-Pascual, P. M., Alloin, D., Clavel, J., et al. 1997, ApJS, 110, 9

Sakata, Y., Minezaki, T., Yoshii, Y., et al. 2010, ApJ, 711, 461

Schlafly, E. F. \& Finkbeiner, D. P. 2011, ApJ, 737, 103

Sergeev, S. G., Pronik, V. I., Peterson, B. M., Sergeeva, E. A., \& Zheng, W. 2002, ApJ, 576, 660

Shakura, N. I. \& Sunyaev, R. A. 1973, A\&A, 500, 33

Shapovalova, A. I., Popović, L. Č., Burenkov, A. N., et al. 2013, A\&A, 559, A10

Shen, Y., Horne, K., Grier, C. J., et al. 2016, ApJ, 818, 30

Stetson, P. B. 1987, PASP, 99, 191

Storchi-Bergmann, T., Schimoia, J. S., Peterson, B. M., et al. 2017, ApJ, 835, 236

Tody, D. 1986, in Society of Photo-Optical Instrumentation Engineers (SPIE) Conference Series, Vol. 627, Instrumentation in astronomy VI, ed. D. L. Crawford, 733

Tody, D. 1993, in Astronomical Society of the Pacific Conference Series, Vol. 52 , Astronomical Data Analysis Software and Systems II, ed. R. J. Hanisch, R. J. V. Brissenden, \& J. Barnes, 173

Ulvestad, J. S. \& Wilson, A. S. 1984, ApJ, 278, 544

Ward, M. J., Wilson, A. S., Penston, M. V., et al. 1978, ApJ, 223, 788

Winkler, H., Glass, I. S., van Wyk, F., et al. 1992, MNRAS, 257, 659

Woo, J.-H. \& Urry, C. M. 2002, ApJ, 579, 530

Wright, E. L. 2006, PASP, 118, 1711

Xiao, M., Du, P., Horne, K., et al. 2018, ApJ, 864, 109

Zetzl, M., Kollatschny, W., Ochmann, M. W., et al. 2018, A\&A, 618, A83 


\section{Appendix A: Additional figures}

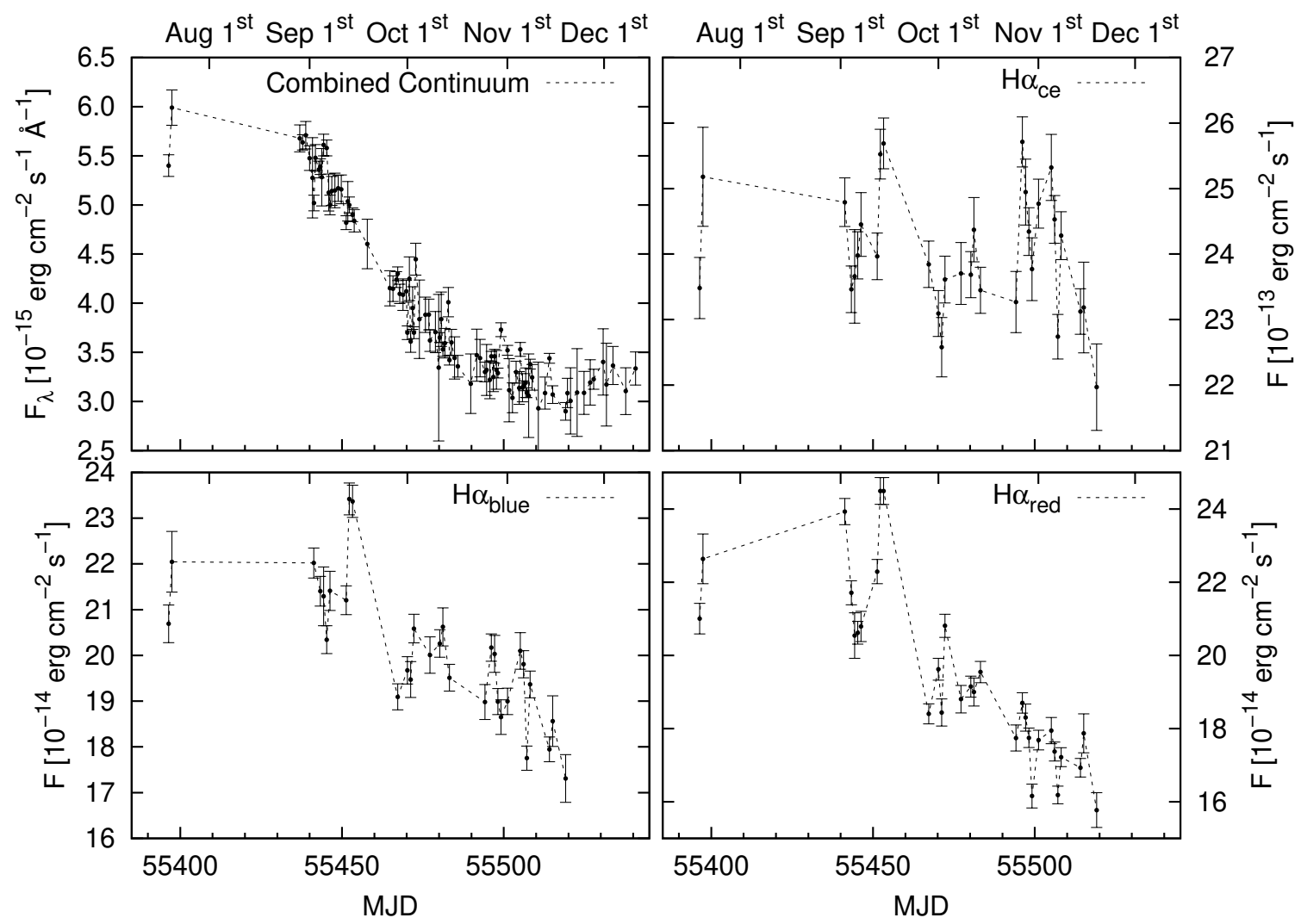

Fig. A.1: Light curves of the combined continuum flux densities at $5420 \AA$ (observed frame; in units of $10^{-15} \mathrm{erg} \mathrm{cm}^{-2} \mathrm{~s}^{-1} \AA^{-1}$ ) as well as of the $\mathrm{H} \alpha$ segment (center, blue, red) light curves.

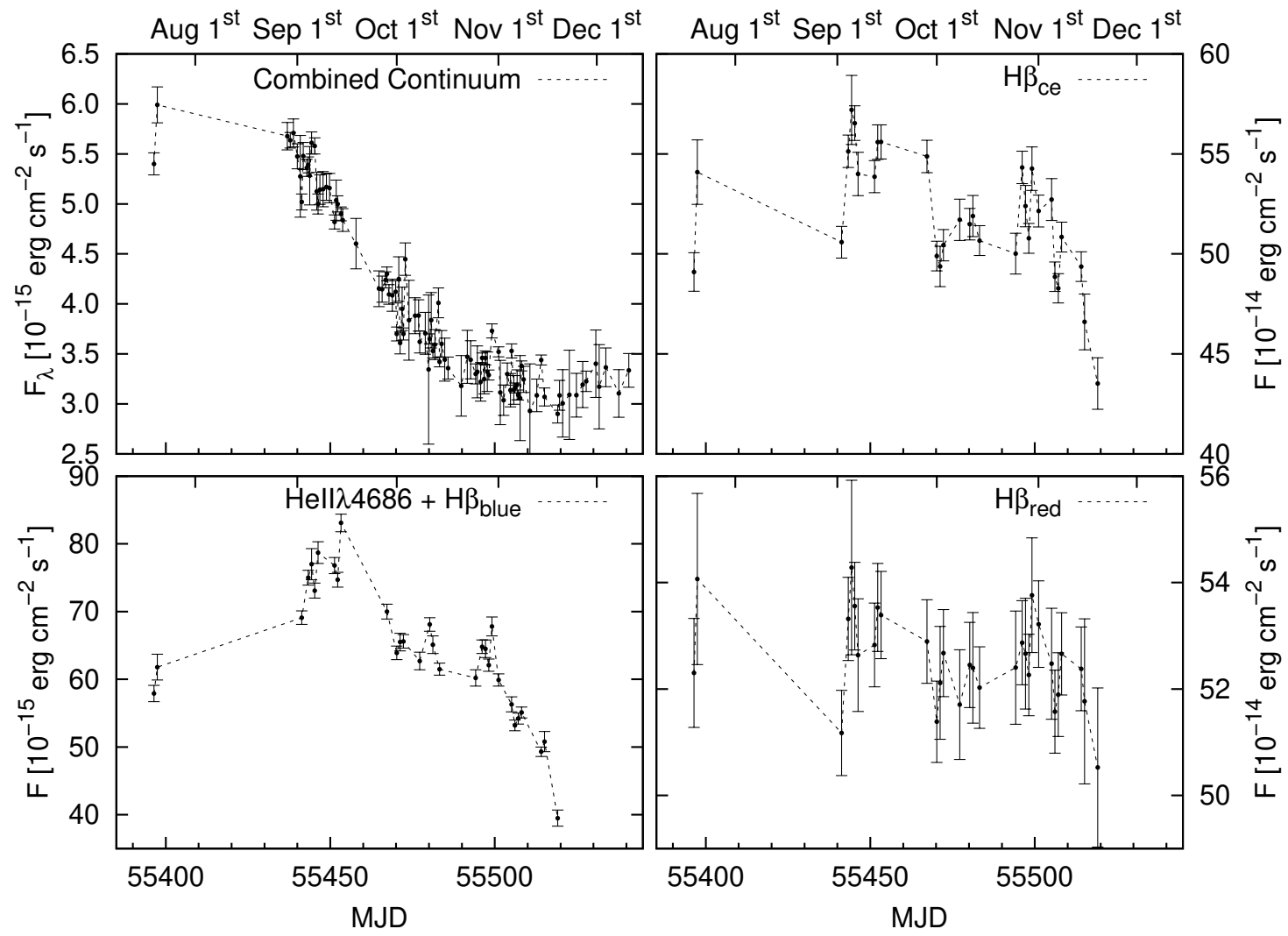

Fig. A.2: Light curves of the combined continuum flux densities at $5420 \AA$ (observed frame; in units of $10^{-15} \mathrm{erg} \mathrm{cm}^{-2} \mathrm{~s}^{-1} \AA^{-1}$ ) as well as of the $\mathrm{H} \beta$ segment (center, blue, red) light curves. 


\section{Appendix B: Additional tables}

Table B.1: HET continuum flux densities (observed frame).

\begin{tabular}{|c|c|c|c|c|c|c|c|}
\hline $\begin{array}{l}\text { Mod. Julian Date } \\
\text { (1) }\end{array}$ & $\begin{array}{c}\text { Cont. } 4425 \AA \\
\text { (2) }\end{array}$ & $\begin{array}{c}\text { Cont. } 4755 \AA \\
\text { (3) }\end{array}$ & $\begin{array}{c}\text { Cont. } 5420 \AA \\
\text { (4) }\end{array}$ & $\begin{array}{c}\text { Cont. } 5485 \AA \\
\text { (5) }\end{array}$ & $\begin{array}{c}\text { Cont. } 5755 \AA \\
(6)\end{array}$ & $\begin{array}{c}\text { Cont. } 6530 \AA \\
\text { (7) }\end{array}$ & $\begin{array}{c}\text { Cont. } 7255 \AA \\
\text { (8) }\end{array}$ \\
\hline 55396.41 & $8.12 \pm 0.16$ & $7.07 \pm 0.14$ & $5.40 \pm 0.11$ & $5.39 \pm 0.11$ & $4.95 \pm 0.10$ & $4.65 \pm 0.09$ & $4.22 \pm 0.08$ \\
\hline 55397.42 & $8.73 \pm 0.26$ & $7.73 \pm 0.23$ & $5.99 \pm 0.18$ & $5.94 \pm 0.18$ & $5.44 \pm 0.16$ & $4.92 \pm 0.15$ & $4.48 \pm 0.13$ \\
\hline 55441.31 & $7.49 \pm 0.11$ & $6.61 \pm 0.10$ & $5.02 \pm 0.08$ & $4.99 \pm 0.07$ & $4.60 \pm 0.07$ & $4.30 \pm 0.06$ & $4.27 \pm 0.06$ \\
\hline 55443.30 & $7.44 \pm 0.11$ & $6.85 \pm 0.10$ & $5.39 \pm 0.08$ & $5.41 \pm 0.08$ & $4.96 \pm 0.07$ & $4.53 \pm 0.07$ & $4.29 \pm 0.06$ \\
\hline 55444.30 & $7.73 \pm 0.15$ & $7.04 \pm 0.14$ & $5.61 \pm 0.11$ & $5.66 \pm 0.11$ & $5.28 \pm 0.11$ & $4.43 \pm 0.09$ & $4.40 \pm 0.09$ \\
\hline 55445.28 & $7.76 \pm 0.12$ & $6.99 \pm 0.10$ & $5.58 \pm 0.08$ & $5.62 \pm 0.08$ & $5.27 \pm 0.08$ & $4.29 \pm 0.06$ & $4.63 \pm 0.07$ \\
\hline 55446.29 & $7.43 \pm 0.15$ & $6.59 \pm 0.13$ & $5.00 \pm 0.10$ & $5.00 \pm 0.10$ & $4.57 \pm 0.09$ & $4.30 \pm 0.09$ & $4.31 \pm 0.09$ \\
\hline 55451.27 & $7.09 \pm 0.11$ & $6.26 \pm 0.09$ & $4.82 \pm 0.07$ & $4.84 \pm 0.07$ & $4.43 \pm 0.07$ & $4.03 \pm 0.06$ & $4.03 \pm 0.06$ \\
\hline 55452.26 & $6.25 \pm 0.09$ & $5.95 \pm 0.09$ & $5.00 \pm 0.08$ & $5.03 \pm 0.08$ & $4.77 \pm 0.07$ & $4.19 \pm 0.06$ & $3.91 \pm 0.06$ \\
\hline 55453.26 & $7.04 \pm 0.11$ & $6.19 \pm 0.09$ & $4.90 \pm 0.07$ & $4.90 \pm 0.07$ & $4.57 \pm 0.07$ & $4.07 \pm 0.06$ & $4.01 \pm 0.06$ \\
\hline 55467.23 & $5.41 \pm 0.08$ & $5.03 \pm 0.08$ & $4.30 \pm 0.07$ & $4.37 \pm 0.07$ & $4.09 \pm 0.06$ & $3.51 \pm 0.05$ & $4.03 \pm 0.06$ \\
\hline 55470.21 & $5.32 \pm 0.08$ & $4.69 \pm 0.07$ & $3.70 \pm 0.07$ & $3.73 \pm 0.06$ & $3.47 \pm 0.05$ & $3.31 \pm 0.05$ & $3.47 \pm 0.05$ \\
\hline 55471.21 & $4.98 \pm 0.15$ & $4.48 \pm 0.13$ & $3.61 \pm 0.11$ & $3.65 \pm 0.11$ & $3.40 \pm 0.10$ & $3.33 \pm 0.10$ & $3.31 \pm 0.10$ \\
\hline 55472.22 & $4.57 \pm 0.07$ & $4.34 \pm 0.07$ & $3.70 \pm 0.06$ & $3.77 \pm 0.06$ & $3.54 \pm 0.05$ & $3.31 \pm 0.05$ & $3.34 \pm 0.05$ \\
\hline 55477.20 & $4.83 \pm 0.14$ & $4.46 \pm 0.13$ & $3.62 \pm 0.11$ & $3.67 \pm 0.11$ & $3.40 \pm 0.10$ & $3.36 \pm 0.10$ & $3.45 \pm 0.10$ \\
\hline 55480.20 & $4.40 \pm 0.07$ & $4.23 \pm 0.06$ & $3.65 \pm 0.05$ & $3.74 \pm 0.06$ & $3.53 \pm 0.05$ & $3.44 \pm 0.05$ & $3.45 \pm 0.05$ \\
\hline 55481.20 & $4.59 \pm 0.09$ & $4.21 \pm 0.08$ & $3.53 \pm 0.07$ & $3.56 \pm 0.07$ & $3.32 \pm 0.07$ & $3.26 \pm 0.07$ & $3.32 \pm 0.07$ \\
\hline 55483.20 & $4.76 \pm 0.07$ & $4.23 \pm 0.06$ & $3.42 \pm 0.05$ & $3.45 \pm 0.05$ & $3.24 \pm 0.05$ & $3.04 \pm 0.05$ & $3.31 \pm 0.05$ \\
\hline 55494.16 & $4.12 \pm 0.12$ & $3.78 \pm 0.11$ & $3.30 \pm 0.10$ & $3.35 \pm 0.10$ & $3.15 \pm 0.09$ & $3.10 \pm 0.09$ & $3.18 \pm 0.10$ \\
\hline 55496.15 & $4.23 \pm 0.06$ & $3.98 \pm 0.06$ & $3.46 \pm 0.05$ & $3.51 \pm 0.05$ & $3.26 \pm 0.05$ & $3.13 \pm 0.05$ & $3.39 \pm 0.05$ \\
\hline & & & & & & $3.26 \pm 0.06$ & $3.31 \pm 0.07$ \\
\hline 55498.17 & $4.28 \pm 0.06$ & $3.88 \pm 0.06$ & $3.29 \pm 0.05$ & $3.35 \pm 0.05$ & $3.16 \pm 0.05$ & $3.03 \pm 0.05$ & $3.31 \pm 0.05$ \\
\hline 55499.16 & $4.36 \pm 0.09$ & $4.18 \pm 0.08$ & $3.73 \pm 0.07$ & $3.84 \pm 0.08$ & $3.62 \pm 0.07$ & $3.42 \pm 0.07$ & $3.48 \pm 0.07$ \\
\hline 55501.14 & $4.07 \pm 0.06$ & $3.89 \pm 0.06$ & $3.52 \pm 0.05$ & $3.60 \pm 0.05$ & $3.45 \pm 0.05$ & $3.02 \pm 0.05$ & $3.46 \pm 0.05$ \\
\hline 55505.12 & $4.26 \pm 0.09$ & $4.03 \pm 0.08$ & $3.53 \pm 0.07$ & $3.57 \pm 0.07$ & $3.41 \pm 0.07$ & $3.12 \pm 0.06$ & $3.34 \pm 0.07$ \\
\hline 55506.12 & $4.05 \pm 0.06$ & $3.72 \pm 0.06$ & $3.16 \pm 0.05$ & $3.18 \pm 0.05$ & $2.96 \pm 0.04$ & $3.07 \pm 0.05$ & $3.10 \pm 0.05$ \\
\hline 55507.11 & $4.27 \pm 0.06$ & $3.81 \pm 0.06$ & $3.09 \pm 0.05$ & $3.14 \pm 0.05$ & $2.92 \pm 0.04$ & $2.86 \pm 0.04$ & $3.04 \pm 0.05$ \\
\hline 55508.11 & $4.13 \pm 0.06$ & $3.92 \pm 0.06$ & $3.38 \pm 0.05$ & $3.46 \pm 0.05$ & $3.25 \pm 0.05$ & $3.13 \pm 0.05$ & $3.31 \pm 0.05$ \\
\hline 55514.12 & $4.26 \pm 0.06$ & $3.93 \pm 0.06$ & $3.44 \pm 0.05$ & $3.52 \pm 0.05$ & $3.34 \pm 0.05$ & $2.86 \pm 0.04$ & $3.32 \pm 0.05$ \\
\hline 55515.12 & $4.01 \pm 0.12$ & $3.57 \pm 0.11$ & $3.07 \pm 0.09$ & $3.12 \pm 0.09$ & $2.91 \pm 0.09$ & $2.89 \pm 0.09$ & $2.95 \pm 0.09$ \\
\hline 55519.10 & $3.93 \pm 0.12$ & $3.52 \pm 0.11$ & $2.90 \pm 0.09$ & $2.96 \pm 0.09$ & $2.72 \pm 0.08$ & $2.76 \pm 0.08$ & $2.96 \pm 0.09$ \\
\hline
\end{tabular}

Notes. Continuum flux densities in units of $10^{-15} \mathrm{erg} \mathrm{s}^{-1} \mathrm{~cm}^{-2} \AA^{-1}$. 
Table B.2: Integrated broad-line fluxes - including the narrow components - of the Balmer and helium lines for different epochs.

\begin{tabular}{|c|c|c|c|c|c|}
\hline $\begin{array}{l}\text { Mod. Julian Date } \\
\text { (1) }\end{array}$ & $\begin{array}{l}\mathrm{H} \alpha \\
(2)\end{array}$ & $\begin{array}{l}\mathrm{H} \beta \\
(3)\end{array}$ & $\begin{array}{l}\mathrm{H} \gamma \\
(4)\end{array}$ & $\begin{array}{l}\text { He } \mathrm{I}_{\text {center }} \\
\quad(5)\end{array}$ & $\begin{array}{c}\text { He II }+H \beta_{\text {blue }} \\
\text { (6) }\end{array}$ \\
\hline 55396.41 & $2765 . \pm 55$ & $1072 . \pm 21$ & $152.7 \pm 3.1$ & $85.4 \pm 2.2$ & $57.9 \pm 1.2$ \\
\hline 55397.42 & $2965 . \pm 89$ & $1143 . \pm 34$ & $163.6 \pm 4.9$ & $93.0 \pm 1.4$ & $61.8 \pm 1.9$ \\
\hline 55441.31 & $2939 . \pm 44$ & $1087 . \pm 17$ & $136.6 \pm 2.1$ & $96.6 \pm 1.5$ & $69.1 \pm 1.0$ \\
\hline 55443.30 & $2777 . \pm 42$ & $1160 . \pm 17$ & $129.6 \pm 1.9$ & $105.0 \pm 2.7$ & $75.0 \pm 1.1$ \\
\hline 55444.30 & $2784 . \pm 84$ & $1192 . \pm 36$ & $129.7 \pm 3.9$ & $115.8 \pm 1.8$ & $77.0 \pm 2.3$ \\
\hline 55445.28 & $2808 . \pm 42$ & 1174. \pm 18 & $147.3 \pm 2.2$ & $109.1 \pm 2.8$ & $73.1 \pm 1.1$ \\
\hline 55446.29 & $2867 . \pm 57$ & $1145 . \pm 23$ & $146.6 \pm 2.9$ & $93.7 \pm 1.5$ & $78.7 \pm 1.6$ \\
\hline 55451.27 & $2832 . \pm 42$ & $1144 . \pm 17$ & $157.6 \pm 2.4$ & $98.8 \pm 2.0$ & $76.8 \pm 1.2$ \\
\hline 55452.26 & $3032 . \pm 45$ & 1166. \pm 18 & $138.2 \pm 2.1$ & $132.2 \pm 2.0$ & $74.7 \pm 1.1$ \\
\hline 55453.26 & $3048 . \pm 46$ & $1173 . \pm 18$ & $160.6 \pm 2.4$ & $121.5 \pm 1.9$ & $83.1 \pm 1.3$ \\
\hline 55467.23 & $2759 . \pm 41$ & $1148 . \pm 17$ & $155.5 \pm 2.3$ & $104.6 \pm 1.6$ & $70.0 \pm 1.1$ \\
\hline 55470.21 & $2702 . \pm 41$ & $1077 . \pm 16$ & $152.4 \pm 2.3$ & $92.4 \pm 1.4$ & $63.9 \pm 1.0$ \\
\hline 55471.21 & $2637 . \pm 53$ & $1080 . \pm 22$ & $156.6 \pm 3.1$ & $99.4 \pm 1.5$ & $65.5 \pm 1.3$ \\
\hline 55472.22 & $2775 . \pm 42$ & $1097 . \pm 17$ & $155.0 \pm 2.3$ & $106.2 \pm 1.6$ & $65.6 \pm 1.0$ \\
\hline 55477.20 & $2759 . \pm 55$ & $1104 . \pm 22$ & $163.5 \pm 3.3$ & $98.8 \pm 2.0$ & $62.7 \pm 1.3$ \\
\hline 55480.20 & $2763 . \pm 41$ & $1115 . \pm 17$ & $164.4 \pm 2.5$ & $105.5 \pm 1.6$ & $68.1 \pm 1.0$ \\
\hline 55481.20 & $2834 . \pm 57$ & $1108 . \pm 22$ & $173.4 \pm 3.5$ & $101.3 \pm 1.6$ & $65.1 \pm 1.3$ \\
\hline 55483.20 & $2735 . \pm 41$ & $1088 . \pm 16$ & $178.4 \pm 2.7$ & $93.7 \pm 1.5$ & $61.5 \pm 0.9$ \\
\hline 55494.16 & $2694 . \pm 54$ & $1084 . \pm 22$ & $164.6 \pm 3.3$ & $89.8 \pm 1.8$ & $60.2 \pm 1.2$ \\
\hline 55496.15 & $2960 . \pm 44$ & 1137. \pm 17 & $183.5 \pm 2.8$ & $92.2 \pm 1.4$ & $64.8 \pm 1.0$ \\
\hline 55497.16 & $2878 . \pm 58$ & $1115 . \pm 22$ & $184.7 \pm 3.7$ & $92.7 \pm 1.9$ & $64.5 \pm 1.3$ \\
\hline 55498.17 & $2802 . \pm 42$ & $1093 . \pm 16$ & $187.8 \pm 2.8$ & $85.0 \pm 1.7$ & $62.1 \pm 0.9$ \\
\hline 55499.16 & $2725 . \pm 55$ & $1148 . \pm 23$ & $192.1 \pm 3.8$ & $93.1 \pm 2.4$ & $67.8 \pm 1.4$ \\
\hline 55501.14 & $2844 . \pm 43$ & $1114 . \pm 17$ & $181.2 \pm 2.7$ & $92.3 \pm 1.4$ & $59.9 \pm 0.9$ \\
\hline 55505.12 & $2913 . \pm 58$ & $1108 . \pm 22$ & $190.3 \pm 3.8$ & $94.7 \pm 2.4$ & $56.3 \pm 1.1$ \\
\hline 55506.12 & $2825 . \pm 42$ & $1058 . \pm 16$ & $166.1 \pm 2.5$ & $85.1 \pm 1.3$ & $53.2 \pm 0.8$ \\
\hline 55507.11 & $2613 . \pm 39$ & 1056. \pm 16 & $180.3 \pm 2.7$ & $73.1 \pm 1.1$ & $54.2 \pm 0.8$ \\
\hline 55508.11 & $2794 . \pm 42$ & $1090 . \pm 16$ & $177.6 \pm 2.7$ & $86.3 \pm 1.3$ & $55.1 \pm 0.8$ \\
\hline 55514.12 & 2661. \pm 40 & 1067. \pm 16 & $159.9 \pm 2.4$ & $85.8 \pm 1.3$ & $49.3 \pm 0.7$ \\
\hline 55515.12 & $2683 . \pm 80$ & $1035 . \pm 31$ & $148.3 \pm 4.4$ & $79.7 \pm 1.2$ & $50.8 \pm 1.5$ \\
\hline 55519.10 & $2528 . \pm 76$ & $980 . \pm 29$ & $143.8 \pm 4.3$ & $66.8 \pm 1.0$ & $39.5 \pm 1.2$ \\
\hline
\end{tabular}

Notes. Line fluxes in units of $10^{-15} \mathrm{erg} \mathrm{s}^{-1} \mathrm{~cm}^{-2}$. 


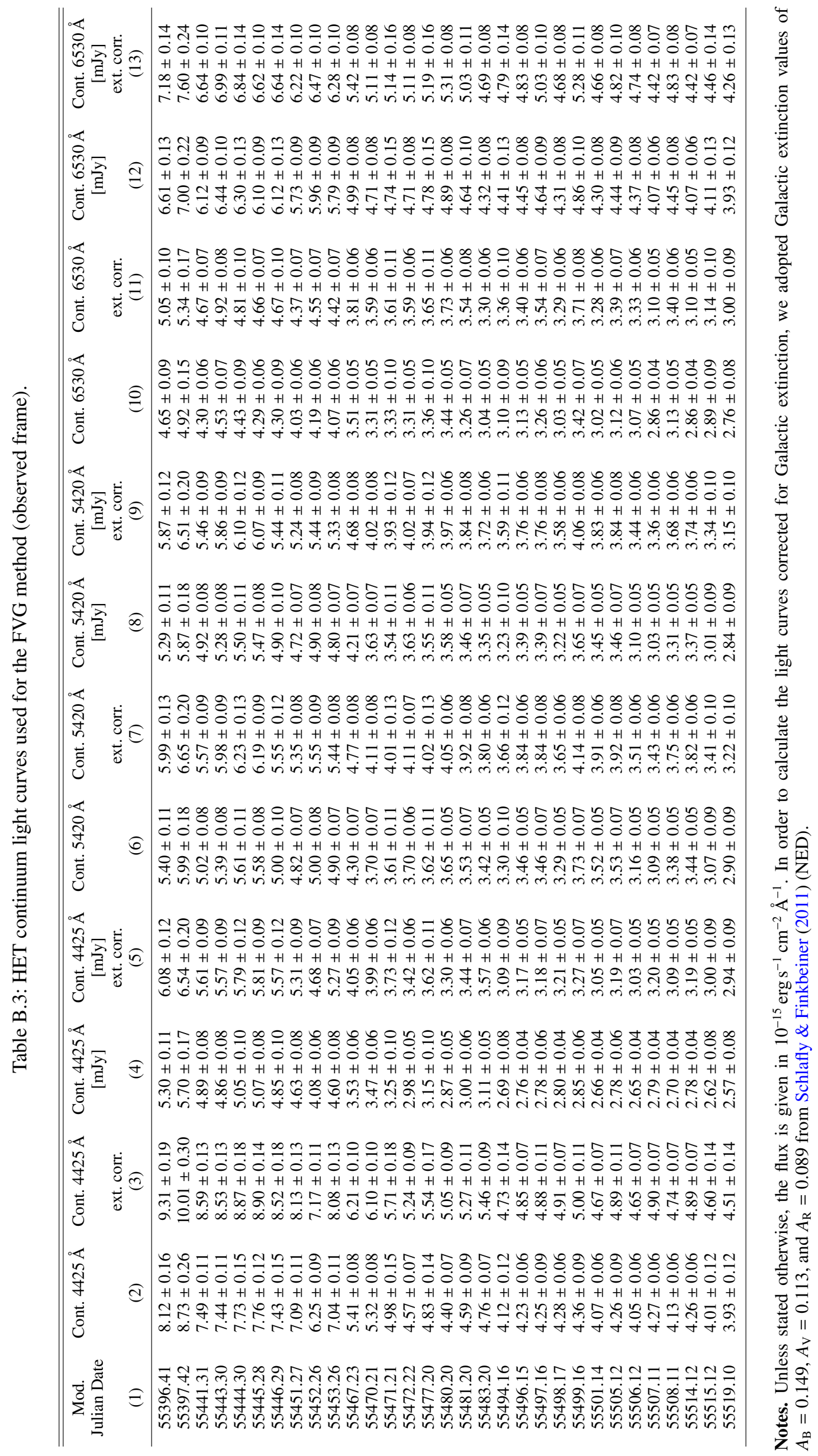


Table B.4: Combined HET $5420 \AA$ (5180 ̊ in rest frame) and Wise Observatory V-band light curve.

\begin{tabular}{|c|c|c|c|c|c|}
\hline $\begin{array}{l}\text { Mod. } \\
\text { Julian Date } \\
\text { (1) }\end{array}$ & $\begin{array}{c}\text { Comb. V band } \\
{\left[10^{-15} \mathrm{erg} \mathrm{s}^{-1} \mathrm{~cm}^{-2} \AA^{-1}\right]} \\
(2)\end{array}$ & $\begin{array}{c}\text { Telescope } \\
\text { (3) }\end{array}$ & $\begin{array}{l}\text { Mod. } \\
\text { Julian Date } \\
\text { (1) }\end{array}$ & $\begin{array}{c}\text { Comb. V band } \\
{\left[10^{-15} \mathrm{erg} \mathrm{s}^{-1} \mathrm{~cm}^{-2} \AA^{-1}\right]} \\
\text { (2) }\end{array}$ & $\begin{array}{c}\text { Telescope } \\
\text { (3) }\end{array}$ \\
\hline 55396.41 & $5.40 \pm 0.11$ & HET & 55481.80 & $3.59 \pm 0.15$ & Wise \\
\hline 55397.42 & $5.99 \pm 0.18$ & HET & 55482.85 & $4.01 \pm 0.15$ & Wise \\
\hline 55436.92 & $5.68 \pm 0.14$ & Wise & 55483.20 & $3.42 \pm 0.05$ & HET \\
\hline 55437.86 & $5.64 \pm 0.08$ & Wise & 55483.82 & $3.60 \pm 0.13$ & Wise \\
\hline 55438.80 & $5.71 \pm 0.14$ & Wise & 55484.80 & $3.44 \pm 0.21$ & Wise \\
\hline 55439.96 & $5.48 \pm 0.13$ & Wise & 55485.81 & $3.36 \pm 0.11$ & Wise \\
\hline 55440.90 & $5.28 \pm 0.41$ & Wise & 55489.79 & $3.18 \pm 0.30$ & Wise \\
\hline 55441.31 & $5.02 \pm 0.08$ & HET & 55491.66 & $3.47 \pm 0.26$ & Wise \\
\hline 55441.80 & $5.48 \pm 0.14$ & Wise & 55492.67 & $3.44 \pm 0.19$ & Wise \\
\hline 55442.88 & $5.36 \pm 0.09$ & Wise & 55494.16 & $3.30 \pm 0.10$ & HET \\
\hline 55443.30 & $5.39 \pm 0.08$ & HET & 55494.67 & $3.32 \pm 0.26$ & Wise \\
\hline 55443.78 & $5.28 \pm 0.29$ & Wise & 55495.68 & $3.22 \pm 0.19$ & Wise \\
\hline 55444.30 & $5.61 \pm 0.11$ & HET & 55496.15 & $3.46 \pm 0.05$ & HET \\
\hline 55445.28 & $5.58 \pm 0.08$ & HET & 55496.75 & $3.25 \pm 0.15$ & Wise \\
\hline 55445.92 & $5.13 \pm 0.19$ & Wise & 55497.16 & $3.46 \pm 0.07$ & HET \\
\hline 55446.29 & $5.00 \pm 0.10$ & HET & 55497.73 & $3.32 \pm 0.20$ & Wise \\
\hline 55446.84 & $5.14 \pm 0.15$ & Wise & 55498.17 & $3.29 \pm 0.05$ & HET \\
\hline 55447.81 & $5.15 \pm 0.18$ & Wise & 55499.16 & $3.73 \pm 0.07$ & HET \\
\hline 55448.81 & $5.17 \pm 0.13$ & Wise & 55501.14 & $3.52 \pm 0.05$ & HET \\
\hline 55449.78 & $5.16 \pm 0.15$ & Wise & 55501.69 & $3.11 \pm 0.32$ & Wise \\
\hline 55451.27 & $4.82 \pm 0.07$ & HET & 55502.71 & $3.04 \pm 0.15$ & Wise \\
\hline 55451.79 & $5.04 \pm 0.20$ & Wise & 55503.79 & $3.30 \pm 0.11$ & Wise \\
\hline 55452.26 & $5.00 \pm 0.08$ & HET & 55504.79 & $3.14 \pm 0.17$ & Wise \\
\hline 55453.26 & $4.90 \pm 0.07$ & HET & 55505.12 & $3.53 \pm 0.07$ & HET \\
\hline 55453.78 & $4.84 \pm 0.11$ & Wise & 55505.76 & $3.14 \pm 0.14$ & Wise \\
\hline 55457.80 & $4.60 \pm 0.25$ & Wise & 55506.12 & $3.16 \pm 0.05$ & HET \\
\hline 55464.79 & $4.15 \pm 0.18$ & Wise & 55506.80 & $3.19 \pm 0.15$ & Wise \\
\hline 55465.76 & $4.15 \pm 0.13$ & Wise & 55507.11 & $3.09 \pm 0.05$ & HET \\
\hline 55466.85 & $4.24 \pm 0.08$ & Wise & 55507.72 & $3.06 \pm 0.43$ & Wise \\
\hline 55467.23 & $4.30 \pm 0.07$ & HET & 55508.11 & $3.38 \pm 0.05$ & HET \\
\hline 55467.84 & $4.09 \pm 0.10$ & Wise & 55508.77 & $3.25 \pm 0.13$ & Wise \\
\hline 55468.83 & $4.09 \pm 0.16$ & Wise & 55510.66 & $2.93 \pm 0.47$ & Wise \\
\hline 55469.84 & $4.12 \pm 0.11$ & Wise & 55512.75 & $3.09 \pm 0.16$ & Wise \\
\hline 55470.21 & $3.70 \pm 0.07$ & HET & 55514.12 & $3.44 \pm 0.05$ & HET \\
\hline 55470.81 & $4.25 \pm 0.22$ & Wise & 55515.12 & $3.07 \pm 0.09$ & HET \\
\hline 55471.21 & $3.61 \pm 0.11$ & HET & 55519.10 & $2.90 \pm 0.09$ & HET \\
\hline 55471.84 & $3.95 \pm 0.22$ & Wise & 55519.69 & $3.09 \pm 0.15$ & Wise \\
\hline 55472.22 & $3.70 \pm 0.06$ & HET & 55520.65 & $3.01 \pm 0.34$ & Wise \\
\hline 55472.80 & $4.45 \pm 0.16$ & Wise & 55522.67 & $3.09 \pm 0.45$ & Wise \\
\hline 55473.92 & $3.84 \pm 0.40$ & Wise & 55524.79 & $3.09 \pm 0.22$ & Wise \\
\hline 55475.76 & $3.88 \pm 0.18$ & Wise & 55526.71 & $3.19 \pm 0.23$ & Wise \\
\hline 55476.85 & $3.88 \pm 0.16$ & Wise & 55527.76 & $3.23 \pm 0.10$ & Wise \\
\hline 55477.20 & $3.62 \pm 0.11$ & HET & 55530.71 & $3.40 \pm 0.34$ & Wise \\
\hline 55478.89 & $3.71 \pm 0.21$ & Wise & 55531.70 & $3.17 \pm 0.42$ & Wise \\
\hline 55479.88 & $3.34 \pm 0.75$ & Wise & 55533.75 & $3.37 \pm 0.19$ & Wise \\
\hline 55480.20 & $3.65 \pm 0.05$ & HET & 55537.67 & $3.11 \pm 0.24$ & Wise \\
\hline 55480.69 & $3.84 \pm 0.28$ & Wise & 55540.72 & $3.34 \pm 0.17$ & Wise \\
\hline 55481.20 & $3.53 \pm 0.07$ & HET & & & \\
\hline
\end{tabular}

